\title{
Trends in Prevalence, Awareness, Treatment, and Control of Diabetes Mellitus in Mainland China from 1979 to 2012
}

\author{
Min-zhi Li, ${ }^{1}$ Li Su, ${ }^{2}$ Bao-yun Liang, ${ }^{1}$ Jin-jing Tan, ${ }^{1}$ Qing Chen, ${ }^{1}$ Jian-xiong Long, \\ Juan-juan Xie, ${ }^{1}$ Guang-liang Wu, ${ }^{1}$ Yan Yan, ${ }^{1}$ Xiao-jing Guo, ${ }^{2}$ and Lian Gu ${ }^{1,3}$ \\ ${ }^{1}$ First Affiliated Hospital, Guangxi University of Chinese Medicine, Nanning, Guangxi 530023, China \\ ${ }^{2}$ School of Public Health of Guangxi Medical University, Nanning, Guangxi 530021, China \\ ${ }^{3}$ Department of Internal Neurology, First Affiliated Hospital, Guangxi University of Chinese Medicine, \\ 89-9 Dongge Road, Nanning, Guangxi, 530023, China \\ Correspondence should be addressed to Lian Gu; gulian2012@163.com
}

Received 17 June 2013; Revised 29 August 2013; Accepted 3 September 2013

Academic Editor: Jack R. Wall

Copyright (c) 2013 Min-zhi Li et al. This is an open access article distributed under the Creative Commons Attribution License, which permits unrestricted use, distribution, and reproduction in any medium, provided the original work is properly cited.

\begin{abstract}
Diabetes mellitus (DM) is one of the primary causes of premature death and disability worldwide. We performed a systematic review and meta-analysis of the published literature regarding the trends in prevalence, awareness, treatment, and control of diabetes mellitus in mainland China. PUBMED, EMBASE, Chinese Biomedical Database, China National Infrastructure database, Chinese Wan Fang database, and Chongqing VIP database were searched. Fifty-six eligible studies were included. Increasing trends in the prevalence, treatment, and control of diabetes in mainland China from 1979 to 2012 were observed. The pooled prevalence, awareness, treatment, and control of diabetes mellitus were $6.41 \%, 45.81 \%, 42.54 \%$, and $20.87 \%$, respectively. A higher prevalence of diabetes mellitus was found in urban $(7.48 \%, 95 \% \mathrm{CI}=5.45 \sim 9.50)$ than rural $(6.53 \%, 95 \% \mathrm{CI}=4.30 \sim 8.76)$ areas. Furthermore, an increasing chronological tendency was shown in different subgroups of age with regard to the prevalence of diabetes. A higher awareness of DM was found in urban $(44.25 \%, 95 \% \mathrm{CI}=32.60 \sim 55.90)$ than rural $(34.27 \%, 95 \% \mathrm{CI}=21.00 \sim 47.54)$ populations, and no significant differences were found in the treatment, and control of diabetes among the subgroups stratified by gender and location. From 1979 to 2012, the prevalence, treatment, and control of diabetes mellitus increased; nevertheless, there was no obvious improvement in the awareness of diabetes.
\end{abstract}

\section{Introduction}

With the rapid economic development, elevated standard of living, dietary shifts, lifestyle alterations, and aging, diabetes mellitus (DM) has become an important public health problem worldwide [1-3], which is estimated to be the third most challenging disease threatening public health after malignant tumors and cardiocerebral vascular diseases [4]. It has been estimated that the global number of individuals with diabetes will double from 171 million in 2000 to 366 million in 2030 among adults aged $\geq 20$ years [5]. Data from European countries have indicated that the health care expenditure for patients with diabetes mellitus was significantly higher than for those who were not diagnosed with this disease [6-8]. Also, in the United States, it has been estimated that approximately 17.5 million people were diagnosed with insulin-dependent diabetes mellitus (IDDM) or non-insulindependent diabetes mellitus (NIDDM) in 2007, and the total annual cost in higher medical costs and lost productivity was estimated to be $\$ 174.4$ billion [9], including \$159.5 billion for the 16.5 million people with NIDDM and $\$ 14.9$ billion for the 1 million people with IDDM [10].

A recent global study indicated that the prevalence of diabetes mellitus was rising rapidly, particularly in developing countries [5]. Moreover, more than $60 \%$ of the population with diabetes mellitus all worldwide comes from Asia, as this remains the world's most populous region. The number of persons living with diabetes will increase substantially in each Asian country over the next few decades [5]. China is the world's largest developing economy and the most populous country, with one-fifth of the global population. Due to the rapid westernization of diet and lifestyle, China has one of the largest diabetes mellitus populations around the world [11]. The total number of people with diabetes in China is projected to increase from 20.8 million in 2000 to 42.3 million 
in 2030 [5]. Diabetes and its complications also result in significant economic burden among individuals, families, and health care systems. According to WHO estimates, China will lose $\$ 558$ billion of its national income to heart disease, stroke, and diabetes from 2006 to 2015 [12]. Therefore, there is an urgent need to reduce the future diabetes burden by providing adequate financial resources and structures of health care delivery in China, particularly within the context of continued rapid urbanization [13].

Diabetes can affect many organ systems throughout the body (e.g., nervous system, renal system, and eyes) and can lead to serious complications over time [14, 15]. Recently, a meta-analysis confirmed that individuals with diabetes mellitus have an approximately 2-fold higher risk of largevessel disease, such as coronary heart disease (CHD) and stroke [16], and nonvascular mortality [17]. Thus, control of the growing prevalence of diabetes mellitus has been widely promoted in order to reduce the risk of large-vessel disease [18]. The pharmacological treatment of diabetes mellitus [19] and lifestyle modification [20] have been shown to decrease the incidence of diabetes mellitus. However, it is very important to first determine the prevalence of diabetes mellitus in the general population, the levels of awareness, treatment, and control of diabetes mellitus. The key elements of effective control include an improvement in the awareness of diabetes mellitus among both health professionals and the general population. Some studies [21-23] have revealed that more than half of the individuals with diabetes mellitus were unaware of the condition. Furthermore, the percentage of patients with diabetes mellitus who were treated and controlled to target levels was substantially low. Moreover, knowing factors associated with the conditions should be useful for health services and public health action in terms of management and prevention. An improvement in the awareness, treatment, and control of diabetes is, therefore, essential for the management and reduction of its prevalence.

Recently, a systematic review on the prevalence of diabetes was published which included 22 cross-sectional studies [24]. However, there was no information provided about the trends in the prevalence of diabetes and the awareness, treatment, and control of the disease. Also, this review only included studies published between 2000 and 2010. To date, there has been no systematic review or meta-analysis on the level of awareness, treatment, and control of diabetes in mainland China. Thus, the purpose of the current paper was to perform a systematic review and meta-analysis of the published literature regarding the prevalence, awareness, treatment, and control of diabetes in mainland China. The objectives of our study were (i) to estimate the trends in prevalence, awareness, treatment, and control of diabetes from 1979 to 2012 in mainland China; and (ii) to estimate the gender, location, and age distributions of patients with diabetes. This systematic review and meta-analysis could provide an overview of the epidemiology of diabetes in the past thirty years in China on the level above the provincial.

\section{Materials and Methods}

2.1. Data Sources and Search Strategy. PUBMED, EMBASE, Chinese Biomedical Database (CBM), CNKI database,
Chinese WanFang Database, and Chongqing VIP database were searched from the date of establishment up to February 2013 using the search terms "chronic disease," "metabolic syndrome," "diabetes," "diabetes mellitus," "prevalence," "epidemiology," "awareness," "treatment," "control," "cross-sectional survey," "longitudinal study" and "China". We also examined the reference lists from the articles identified.

Selection Criteria. All included studies were required to meet the following selection criteria: (i) cross-sectional or longitudinal studies that provided the prevalence, awareness, treatment, and control of diabetes in mainland China; (ii) based on population samples rather than volunteers; and (iii) the study population being representative of the provincial or national population. Exclusion criteria were (1) reviews, editorials, letters, commentaries, or reports; (2) articles repeating data from other articles that were already included; (3) selfreporting data; and (4) studies based on special populations, that is, physical examination crowds, industry, or occupational groups, ethnicity, or age groups.

2.2. Data Extraction. Data were extracted independently by two investigators. Any disagreements were resolved by discussion. We contacted the authors of eligible studies to request further or missing information if needed for subsequent analysis. Data regarding the first author, year of publication, study location, survey date, age range, sampling method, location (urban/rural), diagnostic criteria, diagnosis method, total sample size, total case size, gender distribution, age distribution, and prevalence, awareness, treatment, and control of the diabetes were extracted wherever available. An awareness of DM was considered a self-reported previous diagnosis of DM by a physician or other healthcare practitioner among participants with DM. Treatment of DM was determined as self-reported if taking oral hypoglycemic medications, using insulin, or other nonpharmacological treatments for the management of high glucose levels. The management of fasting plasma glucose (FPG) at levels lower than $7.0 \mathrm{rnmol} / \mathrm{L}(126 \mathrm{mg} / \mathrm{dL})$ in patients of DM in treatment was defined as control of DM.

2.3. Statistical Analysis. STATA software version 11.1 (Stata, College Station, TX, USA) and Review Manager (RevMan) version 5.1 [25] were used to calculate the pooled prevalence, awareness, treatment, and control of diabetes from all of the eligible studies. A summary of the prevalence, awareness, treatment, and control estimates was obtained using randomeffects meta-analysis. Statistical heterogeneity was assessed using the $I^{2}$ statistic [26]. Subgroup analyses included gender, location, and age distribution of diabetes.

\section{Results}

3.1. Results of the Search. We identified 45,947 references through electronic searches of PUBMED $(n=3,541)$, EMBASE $(n=4,013)$, Chinese Biomedical database (CBM) $(n=9,547)$, China National Infrastructure database (CNKI) $(n=8,189)$, Chinese WanFang database $(n=9,165)$, and 
Chongqing VIP database $(n=11,492)$. After excluding duplicates and following examination of titles, abstracts, and full texts, 172 potentially eligible studies were identified. In addition, with a manual search of references, two more studies $[27,28]$ were obtained from the papers. Finally, there were 174 publications included for further analysis. Among these studies, 57 included repetitive data, 54 studies were researched in specific populations, and 7 studies were self-reports of diabetes prevalence. Therefore, we obtained 56 studies in total that were suitable for inclusion in our study [21-23, 2779]. Among the included studies, one study [77] contained survey data for two periods, with the data for 2002-2003 being duplicated in another study [43]. All of the included trials were published from 1980 to 2012.

3.2. Characteristics of the Included Studies. We summarized the characteristics of the 56 included studies which contained 7 nationwide studies and 49 provincial studies conducted in the general Chinese population. Among the included studies, there were 56 concerning the prevalence of diabetes, 10 regarding the awareness of diabetes, 8 involving the treatment of diabetes, and 8 regarding the control of diabetes. A total population of 2,058,243 individuals was investigated, and 120,992 patients with diabetes were detected. The 56 studies were conducted among all of the provinces of mainland China, and included 22 provinces, 4 municipalities, and 5 autonomous regions. Four studies were conducted among all ages, with the age of participants in 9 studies being greater than 30 years old; 3 studies did not provide information regarding the age range investigated. The sampling methods applied in the studies included random sampling, stratified sampling, cluster sampling, multistage-stratified sampling, or combinations of these and general surveys. In terms of diagnostic criteria for diabetes, 7 studies used the American Diabetes Association criteria (ADA) from 1997 or 2009, 31 studies used the criteria of the World Health Organization (WHO) from 1985, 1988, 1995, or 1999, one study used the International Diabetes Federation criteria (IDF) from 2005, and one study was performed according to the criteria outlined in the Lanzhou conference on diabetes in China; however, 12 studies did not provide clear information on the diagnostic criteria employed. Eighteen studies used fasting plasma glucose (FPG) and oral glucose tolerance tests (OGTT) as the methods of diagnosis, 17 studies used FPG alone, 13 studies used OGTT, and one study used FPG, OGTT, and urine glucose tests. The prevalence of diabetes in 56 studies varied from $0.61 \%$ to $20.85 \%$. Levels of awareness, treatment, and control ranged from 28.50 to $62.54 \%, 17.72$ to $92.50 \%$, and 6.86 to $35.87 \%$, respectively (Table 1 ).

3.3. Trends in the Prevalence of Diabetes. As shown in Table 2, the overall prevalence of diabetes was $6.41 \%$ (95\%CI: 5.507.33). The prevalence of diabetes by year varied from $0.81 \%$ to $15.60 \%$. Table 2 and Figure 1(a) illustrate the trend in the overall prevalence of diabetes in mainland China from 1979 to 2012, showing that it increased as time progressed. The lowest prevalence of $0.81 \%$ was found in 1979 initially and showed a stable increase until 2001. After a slight decrease, the prevalence increased quickly from 2002 to 2009 (with the highest prevalence of $15.60 \%$ in 2009), before decreasing rapidly again from 2009 to 2012 .

Forty-three studies reported the prevalence of diabetes by gender. The prevalence of diabetes in males was $6.91 \%$ $(95 \% \mathrm{CI}=5.72-8.09)$, and the prevalence in females was $6.43 \%(95 \% \mathrm{CI}=5.12-7.74)$; there was no significant difference in the prevalence of diabetes between males and females $(\mathrm{OR}=1.07,95 \% \mathrm{CI}=0.98-1.16)$. Overall, trends in the prevalence of diabetes between males and females were increased and similar to the overall trend for the prevalence of diabetes; no significant differences could be observed between males and females (Figure 1(b)).

Fifteen studies provided the prevalence of diabetes by location. The prevalence of diabetes in urban and rural areas was $7.82 \%(95 \% \mathrm{CI}=5.93-9.72)$ and $6.26 \%(95 \% \mathrm{CI}=4.25-$ 8.27), respectively. A statistically significant difference could be found in the prevalence of diabetes between urban and rural areas $(\mathrm{OR}=1.61,95 \% \mathrm{CI}=1.25-2.06)$. As shown in Figure 1(c), trends in the prevalence of diabetes in both urban and rural areas increased over time, and the prevalence of diabetes in urban areas was consistently higher than that in rural areas.

There were 26 articles which reported the prevalence of diabetes stratified by age. The prevalence of diabetes in the groups aged less than 39, 40-59, and over 60 was 1.98\%, $6.96 \%$, and $13.24 \%$, respectively. Table 2 and Figure 1(d) show the information and trends in the prevalence of diabetes by age. An increasing tendency could be observed in all age groups over time. Also, the prevalence of diabetes increased with age (Figure 1(d)).

3.4. Trends in the Awareness of Diabetes. Ten studies provided information on awareness of diabetes. The pooled estimate for the awareness of diabetes was $45.81 \%(95 \% \mathrm{CI}=$ 37.88-53.74) (Table 3). However, no obvious increasing trend could be observed in awareness of diabetes from 1998 to 2012 (Figure 2(a)).

Seven studies reported the awareness of diabetes by gender. Overall, the rate of awareness of diabetes in males was $40.86 \%$ and was $41.58 \%$ for females. No significant difference could be found in the awareness of diabetes between males and females ( $\mathrm{OR}=0.99,95 \% \mathrm{CI}=0.87-1.13)$ (Table 3$)$.

Four studies reported the awareness rate of diabetes for urban and rural populations. The awareness rate in urban areas was $44.25 \%(95 \% \mathrm{CI}=32.6-55.9)$, while it was $34.27 \%$ $(95 \% \mathrm{CI}=21-47.54)$ in rural locations (Table 3$)$.

3.5. Trend in the Treatment of Diabetes. The combined result for the treatment rate of diabetes was $42.54 \%$ (95\% CI $=13.69$ 71.38) (Table 3). As shown in Figure 2(b), an increasing tendency in the treatment of diabetes could be observed from 2001 to 2005 . However, it decreased quickly after 2008 and then rapidly increased again from 2010 to 2011 .

Six studies provided the treatment rate of diabetes stratified by gender. The treatment rate of diabetes in males was $38.48 \%$, whereas it was $41.18 \%$ for females. Comparing the treatment rate of diabetes for males to that of females, no 


\begin{tabular}{|c|c|c|c|c|c|c|c|c|c|c|c|c|c|c|c|c|}
\hline 过 & - & - & - & - & - & - & - & - & - & - & - & - & - & - & & भे \\
\hline 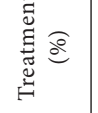 & - & - & - & - & - & - & - & - & - & - & - & - & - & - & - & 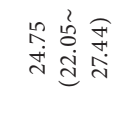 \\
\hline 离 & - & - & - & - & - & - & - & - & - & - & - & - & - & 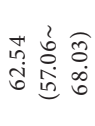 & - & 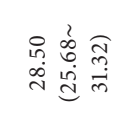 \\
\hline 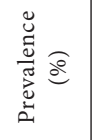 & 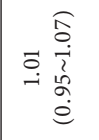 & 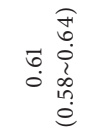 & 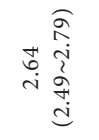 & 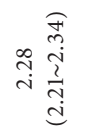 & 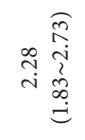 & 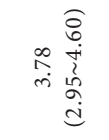 & ro & 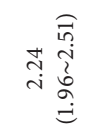 & 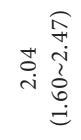 & 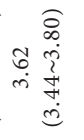 & 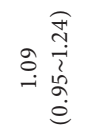 & 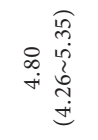 & 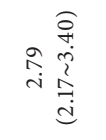 & 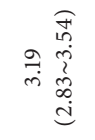 & 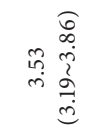 & $\begin{aligned} & 0 \\
& 0 \\
& 0 \\
& 0 \\
& 0 \\
& 0 \\
& 0 \\
& 0 \\
& 0 \\
& 0 \\
& 0\end{aligned}$ \\
\hline 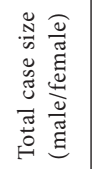 & 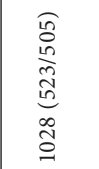 & 点 & $\begin{array}{l}\widehat{ल} \\
\hat{\infty} \\
\infty \\
\infty \\
\stackrel{\infty}{\infty} \\
\stackrel{\infty}{=}\end{array}$ & 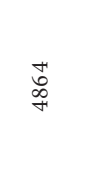 & 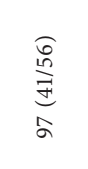 & 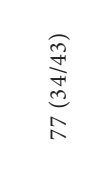 & 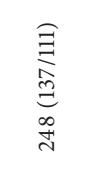 & 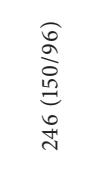 & $\infty$ & 离 & $\overrightarrow{\mathrm{J}}$ & 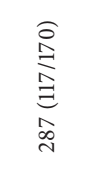 & 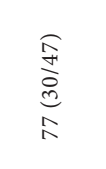 & $\begin{array}{l}\widehat{0} \\
\stackrel{0}{\infty} \\
\stackrel{0}{0} \\
\text { aे }\end{array}$ & 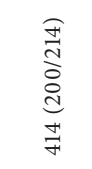 & 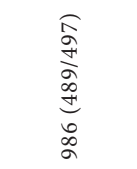 \\
\hline 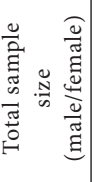 & 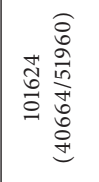 & 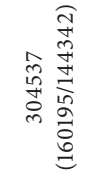 & 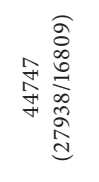 & $\begin{array}{l}\frac{n}{0} \\
\stackrel{n}{\sim} \\
\vec{n}\end{array}$ & 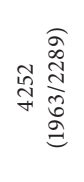 & $\begin{array}{l}\text { के } \\
\text { के } \\
\text { dे } \\
\text { oे } \\
\text { ồ } \\
\text { ते }\end{array}$ & 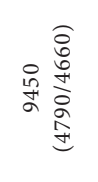 & 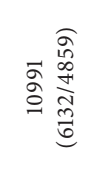 & 点 & $\begin{array}{l}\vec{n} \\
\stackrel{5}{7}\end{array}$ & $\begin{array}{l}\infty \\
\text { đ̃ }\end{array}$ & 突 & 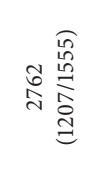 & 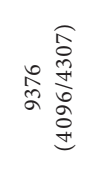 & 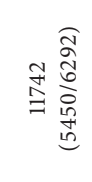 & 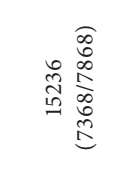 \\
\hline 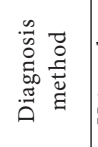 & 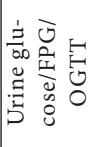 & 旨 & $\begin{array}{l}\text { E } \\
\text { 品 }\end{array}$ & $\begin{array}{l}\vec{E} \\
\dot{E}\end{array}$ & $\begin{array}{l}\dot{E} \\
\dot{0}\end{array}$ & 茾 & $\begin{array}{l}E \\
\dot{E} \\
0\end{array}$ & $\begin{array}{l}E \\
\dot{E} \\
O\end{array}$ & $\vec{\xi}$ & 崖 & $\begin{array}{l}\vec{E} \\
\vec{O}\end{array}$ & 虽 & 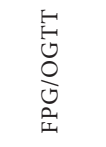 & $\begin{array}{l}\mid \overrightarrow{0} \\
\text { D } \\
0 \\
0 \\
0 \\
0\end{array}$ & 䴕 & \\
\hline 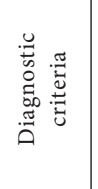 & $\overleftrightarrow{z}$ & 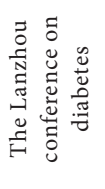 & $\begin{array}{l}\infty \\
\infty \\
0 \\
0 \\
0 \\
3\end{array}$ & 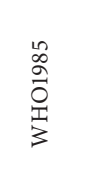 & $\overleftrightarrow{z}$ & 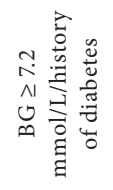 & $\begin{array}{l}\infty \\
\infty \\
0 \\
0 \\
0 \\
3\end{array}$ & $\begin{array}{l}\infty \\
0 \\
0 \\
0 \\
0 \\
3 \\
3\end{array}$ & 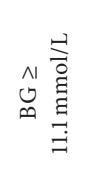 & $\begin{array}{l}\infty \\
\infty \\
2 \\
0 \\
0 \\
3\end{array}$ & $\begin{array}{l}0 \\
\text { 章 } \\
3\end{array}$ & 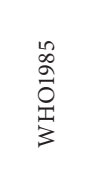 & $\overleftrightarrow{z}$ & 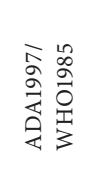 & $\begin{array}{l}\infty \\
0 \\
0 \\
0 \\
0 \\
3\end{array}$ & हे \\
\hline 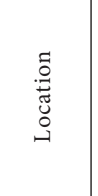 & 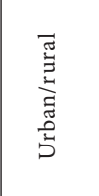 & 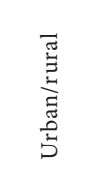 & 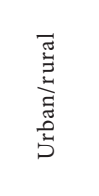 & 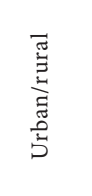 & 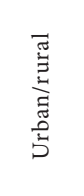 & 宽 & 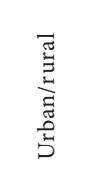 & 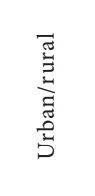 & 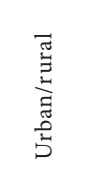 & 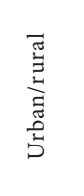 & 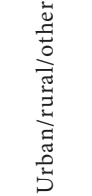 & 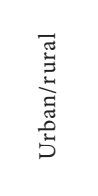 & 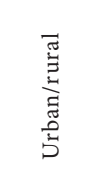 & $\begin{array}{l}\overline{\tilde{E}} \\
\text { 苛 }\end{array}$ & 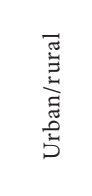 & 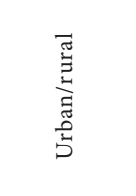 \\
\hline 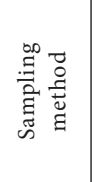 & $\overleftrightarrow{z}$ & 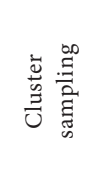 & 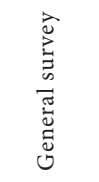 & 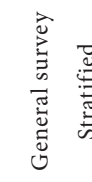 & 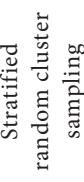 & 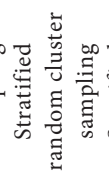 & 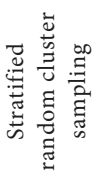 & 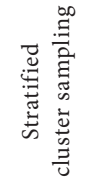 & $\vec{z}$ & 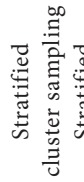 & 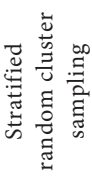 & 8 & 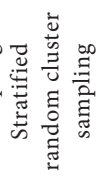 & 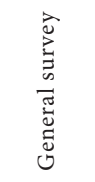 & 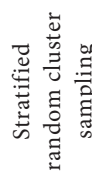 & 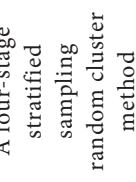 \\
\hline 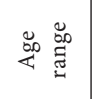 & $\begin{array}{l}\infty \\
0 \\
\vdots \\
0\end{array}$ & 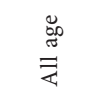 & ؛े̀ & 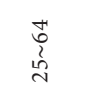 & $\stackrel{2}{2}$ & $\overbrace{n}^{2}$ & 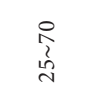 & $\begin{array}{l}\infty \\
\infty \\
⺊ \\
\qquad\end{array}$ & $\stackrel{2}{2}$ & 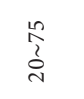 & $\begin{array}{l}\hat{a} \\
\hat{o}\end{array}$ & $\begin{array}{l}\text { + } \\
\stackrel{\sim}{2} \\
\text { }\end{array}$ & 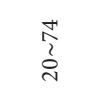 & $\begin{array}{l}\hat{i} \\
\vdots \\
\tilde{n}\end{array}$ & $\begin{array}{l}\text { ते } \\
\text { ते }\end{array}$ & 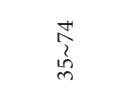 \\
\hline 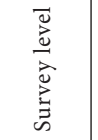 & 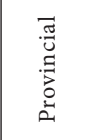 & 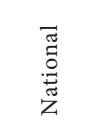 & 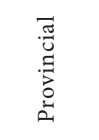 & 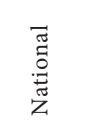 & 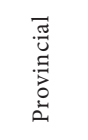 & 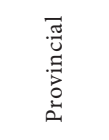 & 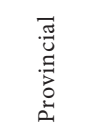 & 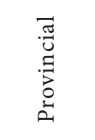 & 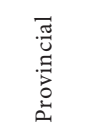 & 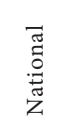 & 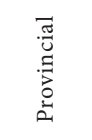 & 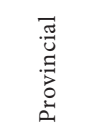 & 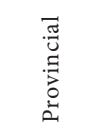 & 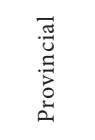 & 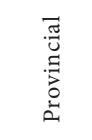 & $\stackrel{\Xi}{\Xi}$ \\
\hline 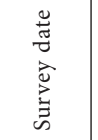 & 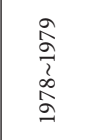 & $\begin{array}{l}\hat{a} \\
\hat{a} \\
\dot{\Delta}\end{array}$ & 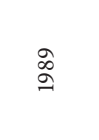 & $\begin{array}{l}\text { 落 } \\
\text { 荌 }\end{array}$ & 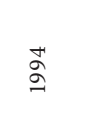 & $\stackrel{n}{\Omega}$ & 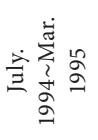 & 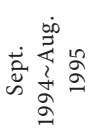 & $\stackrel{\text { ڤn }}{\Omega}$ & 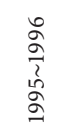 & $\begin{array}{l}0 \\
2 \\
\frac{2}{3} \\
2 \\
2\end{array}$ & 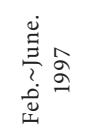 & 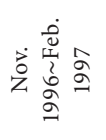 & $\begin{array}{l}\infty \\
\stackrel{\alpha}{g}\end{array}$ & $\begin{array}{l}\infty \\
2 \\
2 \\
2 \\
2 \\
2\end{array}$ & $\frac{2}{8}$ \\
\hline 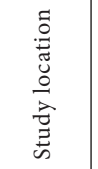 & 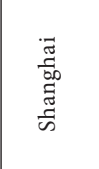 & 胥 & 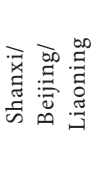 & $\frac{\pi}{\tilde{J}}$ & 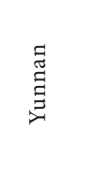 & 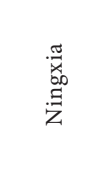 & 矛 & 全 & 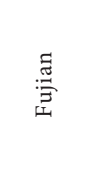 & 栉 & 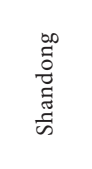 & 苞 & 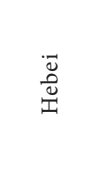 & 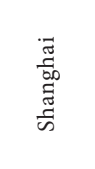 & 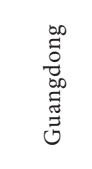 & $\cong$ \\
\hline 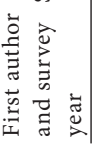 & 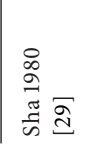 & 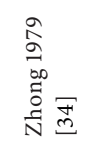 & 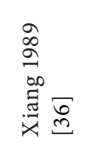 & 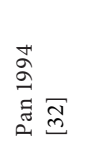 & 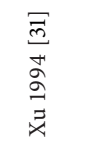 & 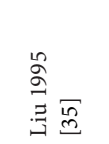 & 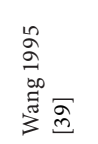 & 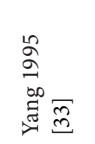 & 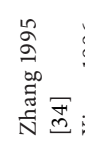 & 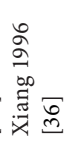 & $\begin{array}{l}\stackrel{0}{\circ} \\
\stackrel{2}{0} \\
\frac{\tilde{J}}{N} \infty \\
\end{array}$ & $\begin{array}{l}\text { Oे } \\
\stackrel{\Xi}{\Xi} \bar{g}\end{array}$ & 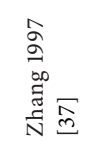 & 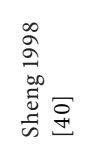 & 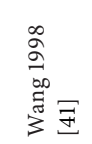 & \\
\hline
\end{tabular}




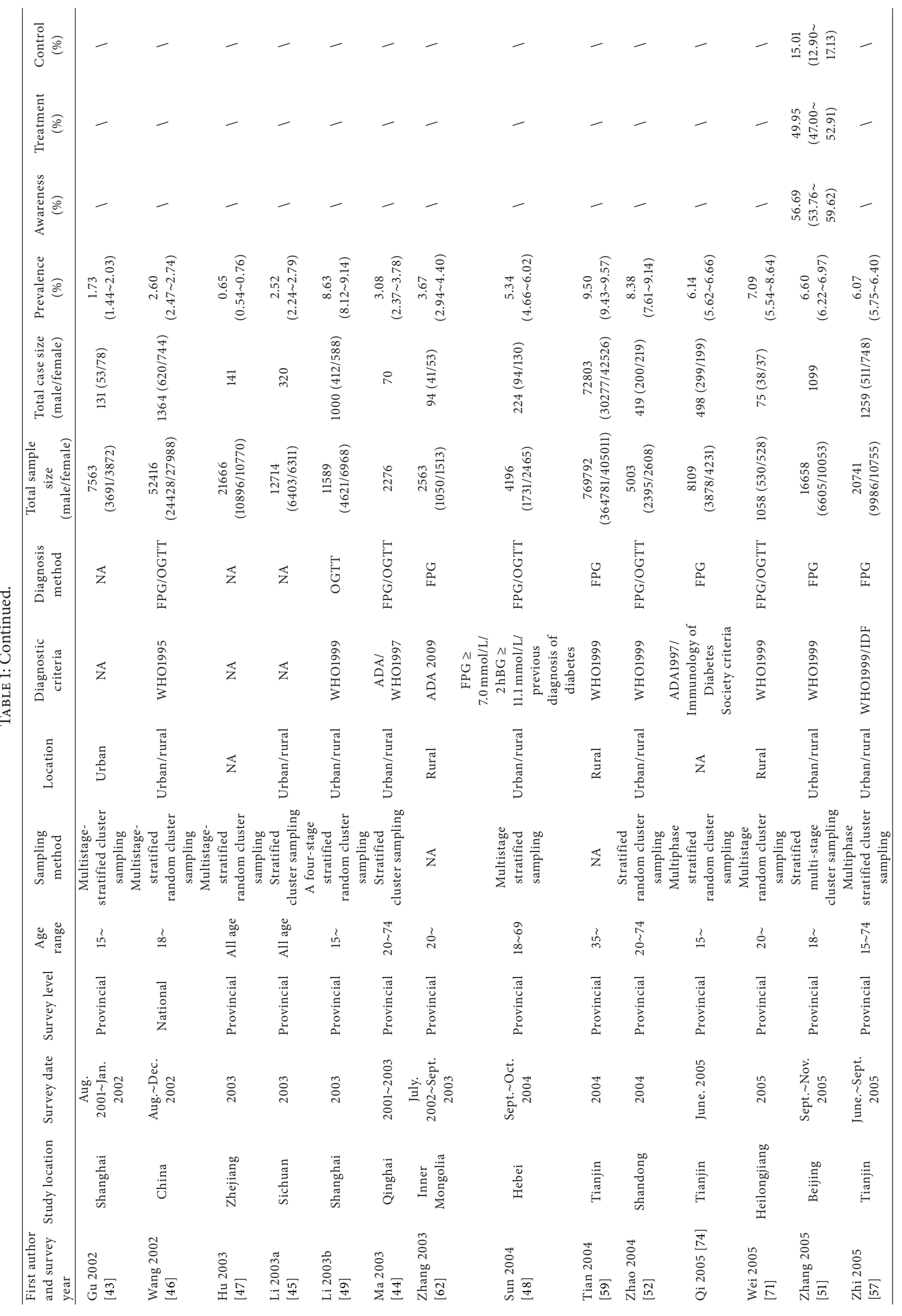




\begin{tabular}{|c|c|c|c|c|c|c|c|c|c|c|c|c|c|c|c|}
\hline 产 & - & - & - & - & - & - & - & 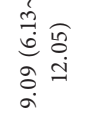 & - & - & - & 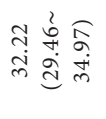 & - & - & 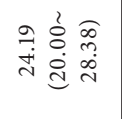 \\
\hline 苞 & - & - & - & - & - & - & $-\frac{7}{7}$ & 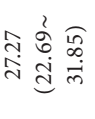 & - & - & - & 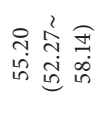 & - & - & 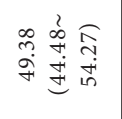 \\
\hline 总 & - & - & - & - & - & - & $-\stackrel{d}{0}$ & 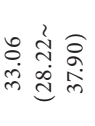 & - & - & - & 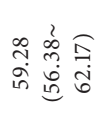 & - & - & 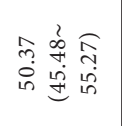 \\
\hline 苛 & 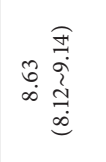 & 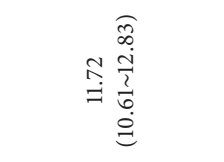 & 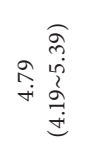 & 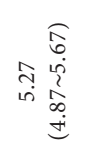 & 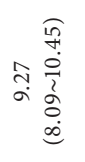 & 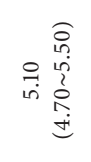 & 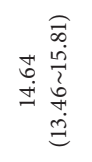 & 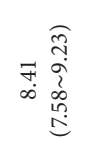 & 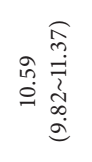 & 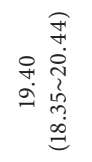 & 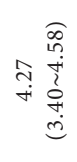 & 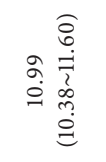 & 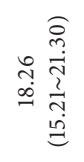 & $\begin{array}{rl} & 0 \\
& 0 \\
0 & 0 \\
0 & 0 \\
\infty & 0 \\
0 & 0 \\
0 & 0\end{array}$ & 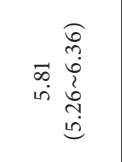 \\
\hline 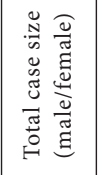 & 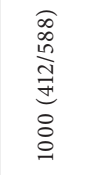 & $\vec{\infty}$ & $\stackrel{\curvearrowright}{\curvearrowright}$ & 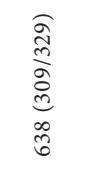 & 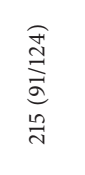 & 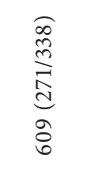 & 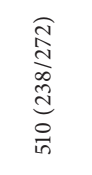 & 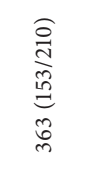 & $\begin{array}{l}\text { a } \\
\frac{7}{a} \\
\text { did } \\
\text { ò }\end{array}$ & $\stackrel{8}{\stackrel{\circ}{\circ}}$ & 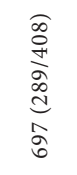 & 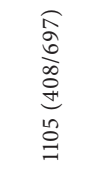 & $\stackrel{9}{\exists}$ & 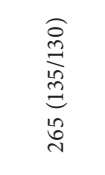 & 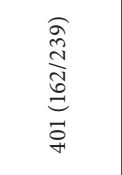 \\
\hline 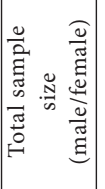 & 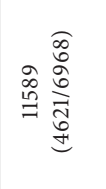 & 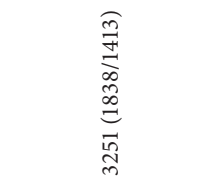 & 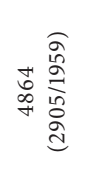 & 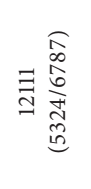 & 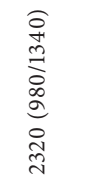 & 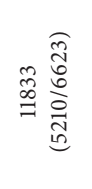 & 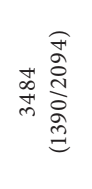 & 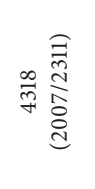 & 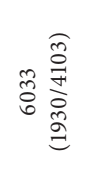 & 总 & 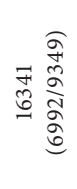 & 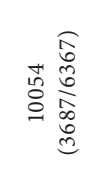 & $\begin{array}{l}\widehat{\widehat{\alpha}} \\
\stackrel{0}{2} \\
\stackrel{\widetilde{d}}{0} \\
\sigma\end{array}$ & 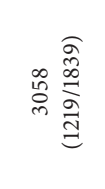 & 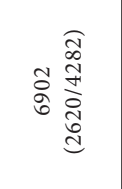 \\
\hline 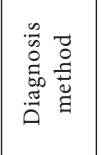 & E & 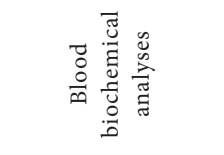 & 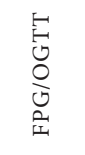 & 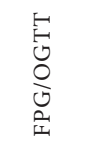 & $\overleftrightarrow{z}$ & 茎 & 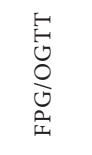 & 茎 & 莺 & 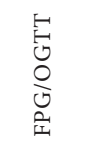 & 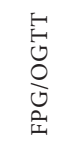 & 望 & 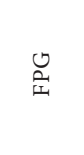 & $\begin{array}{l}\text { 旨 } \\
0 \\
0 \\
0 \\
0 \\
\text { 至 }\end{array}$ & 虽 \\
\hline 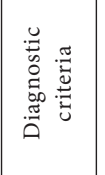 & $\begin{array}{l}\text { Oे } \\
\text { oे } \\
0 \\
\text { Tे } \\
3\end{array}$ & 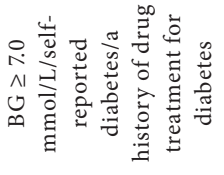 & $\begin{array}{l}2 \\
2 \\
0 \\
0 \\
3 \\
3\end{array}$ & $\begin{array}{l}\text { बे } \\
\text { बे } \\
\text { 苦 } \\
\text { 3 }\end{array}$ & $\overleftrightarrow{z}$ & 呆 & $\begin{array}{l}\text { aे } \\
\text { Oे } \\
\text { 弯 } \\
3\end{array}$ & $\overleftrightarrow{z}$ & $\begin{array}{l}\text { aे } \\
\text { oे } \\
0 \\
\text { 零 }\end{array}$ & 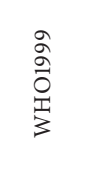 & 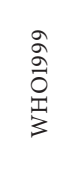 & 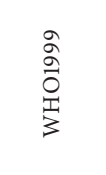 & $\mathbb{z}$ & $\begin{array}{l}\text { o } \\
\text { ò } \\
0 \\
0 \\
3\end{array}$ & 䒚 \\
\hline 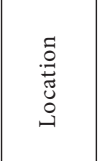 & 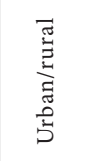 & 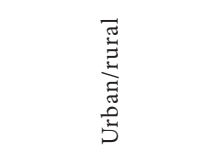 & 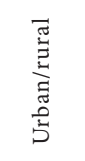 & 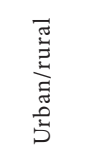 & 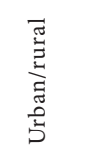 & 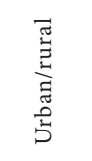 & 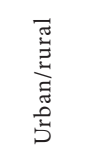 & 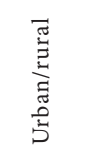 & 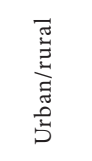 & 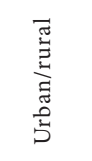 & 㞼 & 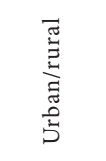 & $\begin{array}{l}\text { 离 } \\
\text { 菅 }\end{array}$ & 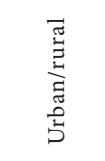 & 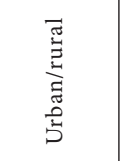 \\
\hline 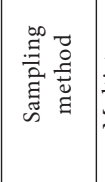 & 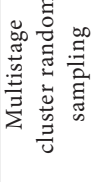 & $\overleftrightarrow{Z}$ & $\overleftrightarrow{z}$ & 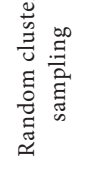 & . & 跣 & & 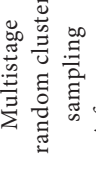 & & & & 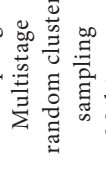 & & 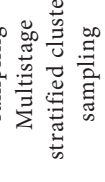 & 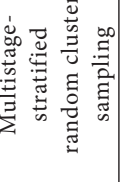 \\
\hline 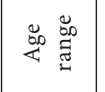 & $\begin{array}{l}\stackrel{+}{2} \\
2 \\
\underline{n}\end{array}$ & 字 & $\overleftrightarrow{z}$ & 2. & $\stackrel{2}{\infty}$ & $\stackrel{2}{\infty}$ & $\stackrel{2}{\text { ¿े }}$ & $\begin{array}{l}0 \\
0 \\
\vdots \\
\end{array}$ & ঐे & ڤे & 究 & $\stackrel{2}{\sim}$ & $\stackrel{2}{a}$ & 芳 & $\iota^{2}$ \\
\hline 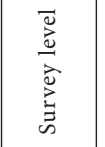 & 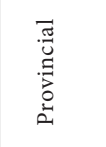 & 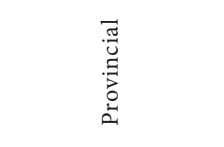 & 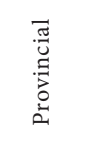 & 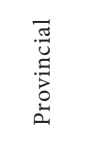 & 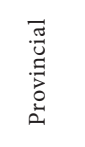 & 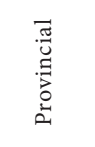 & 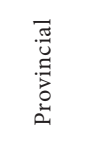 & 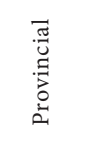 & 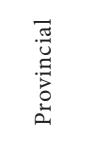 & 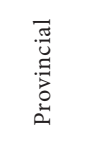 & 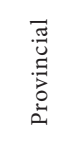 & 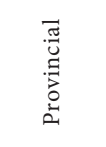 & 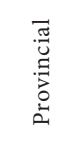 & 呇 & 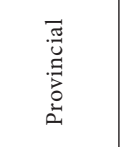 \\
\hline 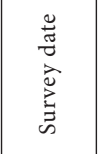 & ঃั & 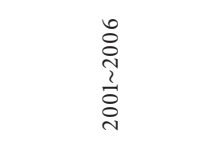 & ঃั & 至 & 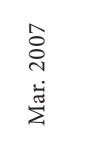 & $\hat{\stackrel{\sim}{े}}$ & 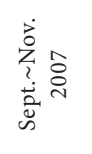 & 今े & $\begin{array}{l}\hat{O} \\
\text { Oे } \\
\text { ?े } \\
\text { ¿ें }\end{array}$ & 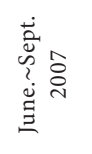 & 今े & 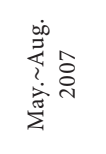 & 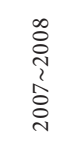 & 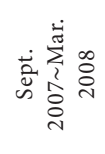 & z \\
\hline 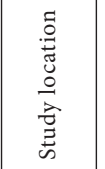 & $\begin{array}{l}\frac{\vec{\pi}}{b} \\
\frac{0}{b 0} \\
\frac{\sigma}{\omega}\end{array}$ & $\stackrel{\infty}{:}$ & 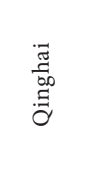 & 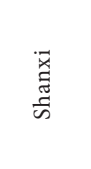 & 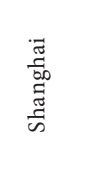 & 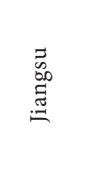 & 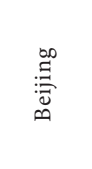 & 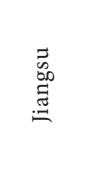 & 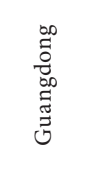 & $\stackrel{\substack{\Xi \\
\Xi}}{\infty}$ & 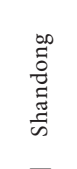 & 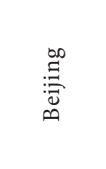 & $\begin{array}{l}\vec{z} \\
\frac{\pi}{\infty} \\
\bar{\sigma} \\
\frac{\pi}{\infty}\end{array}$ & 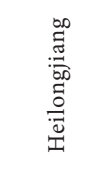 & 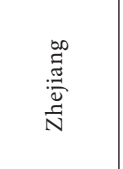 \\
\hline 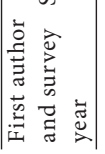 & 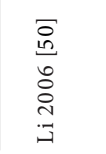 & 这 & 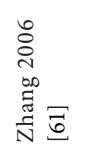 & 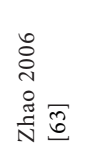 & 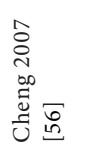 & 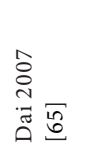 & 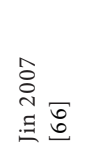 & 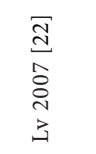 & 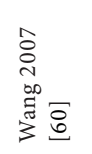 & 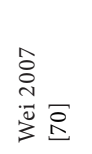 & 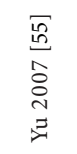 & 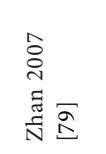 & 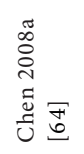 & 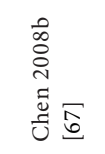 & 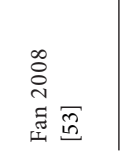 \\
\hline
\end{tabular}




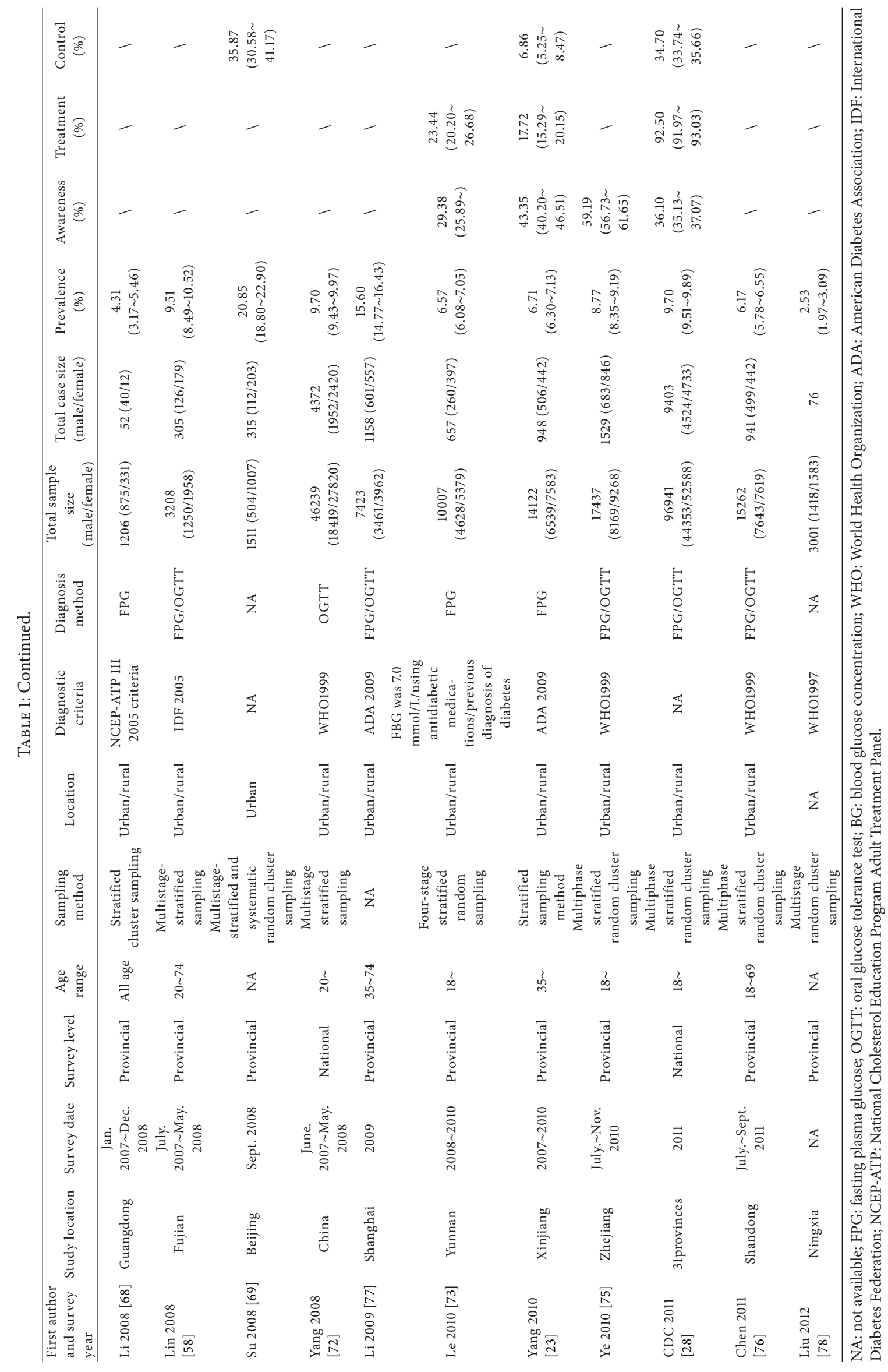




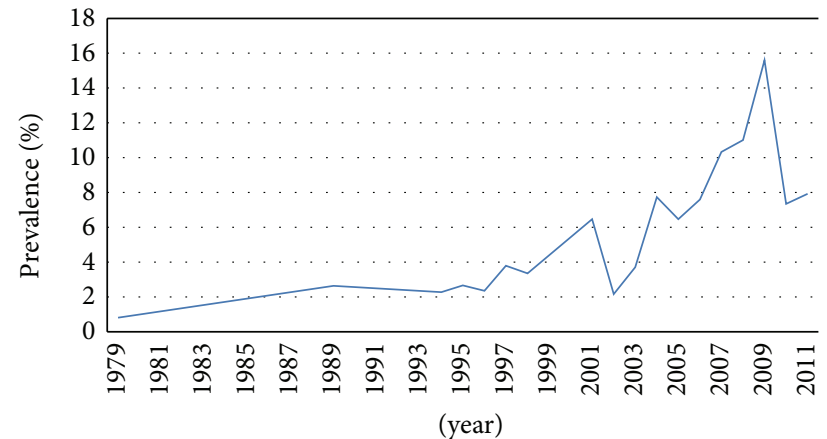

(a)

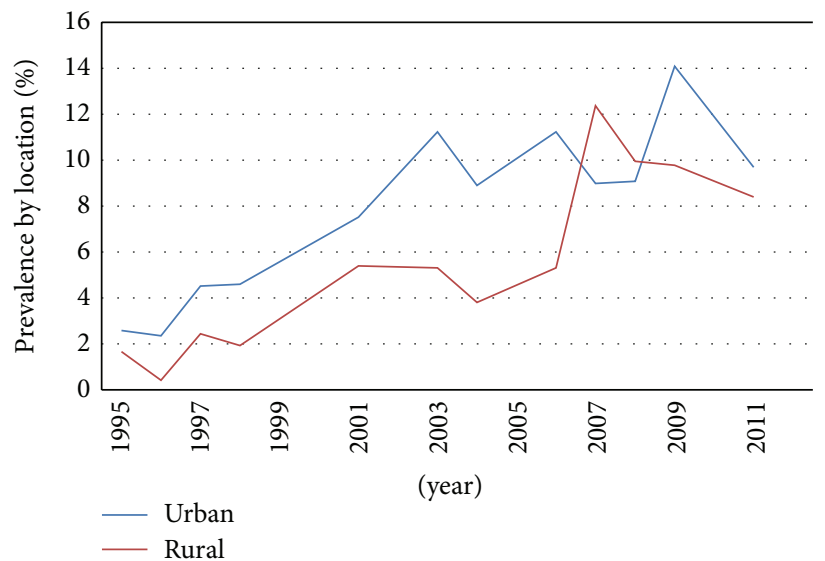

(c)

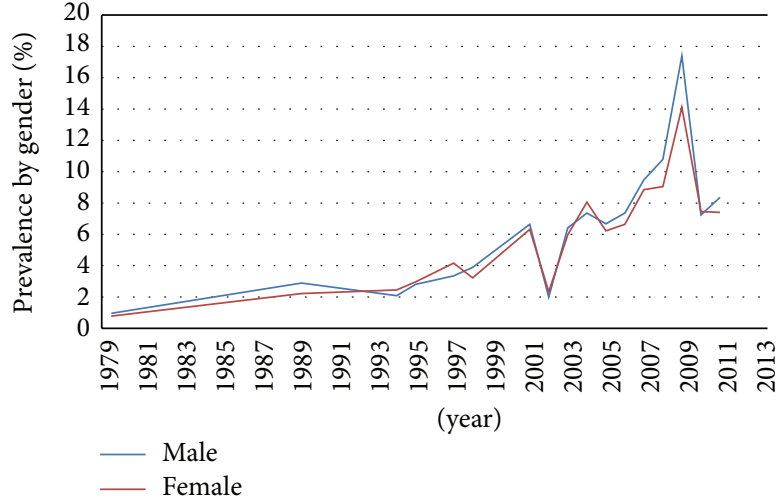

(b)

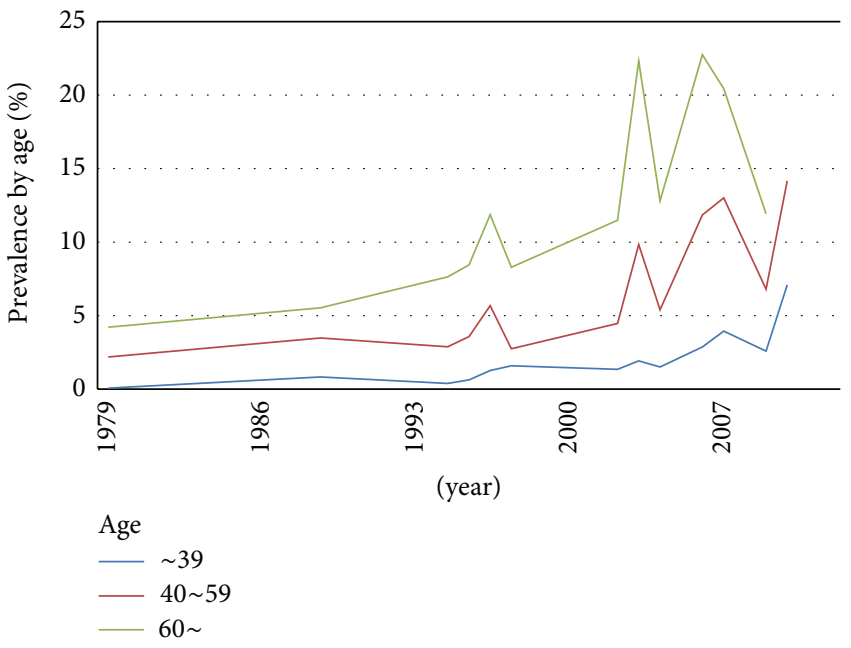

(d)

FIGURE 1: Trends in prevalence of diabetes and subgroup analysis by gender, location, and age.

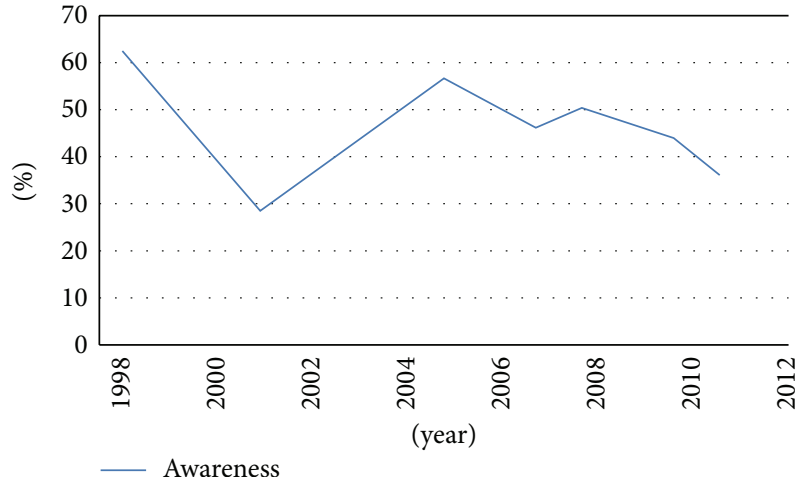

(a)

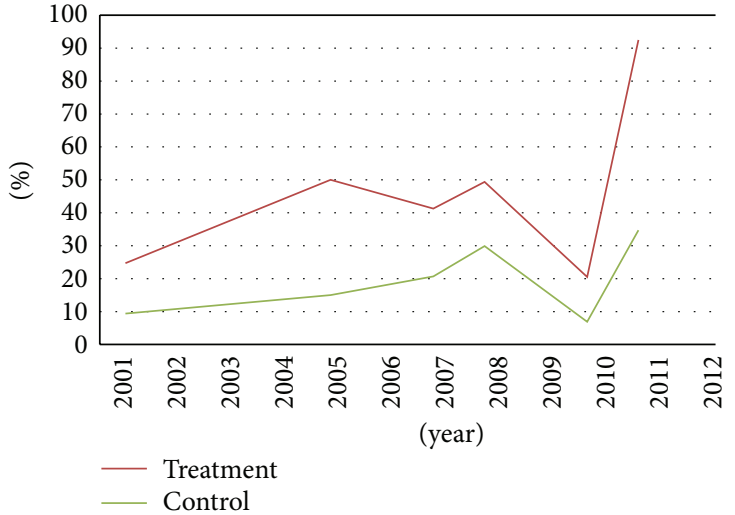

(b)

FIGURE 2: Trends in awareness, treatment, and control of diabetes.

significant difference could be observed $(\mathrm{OR}=0.9,95 \% \mathrm{CI}=$ 0.72-1.14) (Table 3).

Three studies reported the treatment rate by location. The treatment rates in urban and rural areas were 50.18\% $(95 \% \mathrm{CI}=31.22-100.39 \%)$ and $45.42 \%(95 \% \mathrm{CI}=-12.7 \%$ and $-103.6 \%$ ), respectively (Table 3 ). Comparing the treatment rate of diabetes between rural and urban areas, there was no significant difference $(\mathrm{OR}=1.30,95 \% \mathrm{CI}=0.77-2.20)$.

3.6. Trends in the Control of Diabetes. The pooled control rate was $20.87 \%(95 \% \mathrm{CI}=10.76-30.97)$ for diabetes from the combined information of eight studies (Table 3). There was 
TABLE 2: Trends in prevalence of diabetes in mainland China: 1979 2012.

\begin{tabular}{|c|c|c|c|c|c|c|c|c|c|c|c|c|c|c|c|c|c|c|c|}
\hline Survey year & 1979 & 1989 & 1994 & 1995 & 1996 & 1997 & 1998 & 2001 & 2002 & 2003 & 2004 & 2005 & 2006 & 2007 & 2008 & 2009 & 2010 & 2011 & 2012 \\
\hline $\begin{array}{l}\text { Overall } \\
\text { prevalence/ } I^{2}(\%)\end{array}$ & \multicolumn{19}{|c|}{$6.41 / 99.8$} \\
\hline $\begin{array}{l}\text { Prevalence by year } \\
(\%)\end{array}$ & 0.81 & 2.64 & 2.28 & 2.67 & 2.36 & 3.80 & 3.36 & 6.47 & 2.17 & 3.71 & 7.74 & 6.47 & 7.60 & 10.33 & 11.01 & 15.60 & 7.35 & 7.93 & 2.53 \\
\hline \multicolumn{20}{|l|}{ Gender (\%) } \\
\hline Male & 0.96 & 2.89 & 2.09 & 2.81 & 1 & 3.35 & 3.89 & 6.64 & 1.99 & 6.41 & 7.36 & 6.67 & 7.36 & 9.48 & 10.79 & 17.39 & 7.24 & 8.36 & 1 \\
\hline Female & 0.78 & 2.22 & 2.45 & 2.97 & 1 & 4.16 & 3.22 & 6.32 & 2.34 & 5.97 & 8.06 & 6.22 & 6.64 & 8.85 & 9.05 & 14.11 & 7.45 & 7.40 & 1 \\
\hline \multicolumn{20}{|l|}{ Location (\%) } \\
\hline Urban & 1 & 1 & 1 & 2.58 & 2.35 & 4.52 & 4.60 & 7.52 & 1 & 11.23 & 8.90 & 1 & 11.23 & 8.99 & 9.08 & 14.09 & 1 & 9.69 & 1 \\
\hline Rural & 1 & 1 & 1 & 1.66 & 0.42 & 2.44 & 1.93 & 5.40 & 1 & 5.31 & 3.81 & 1 & 5.31 & 12.37 & 9.95 & 9.78 & 1 & 8.40 & 1 \\
\hline \multicolumn{20}{|l|}{ Age (\%) } \\
\hline$\sim 39$ & 0.06 & 0.83 & 1 & 0.39 & 0.63 & 1.27 & 1.59 & 1 & 1 & 1.35 & 1.92 & 1.51 & & 2.87 & 3.94 & & 2.59 & 7.09 & \\
\hline $40 \sim 59$ & 2.19 & 3.48 & 1 & 2.88 & 3.57 & 5.68 & 2.74 & 1 & 1 & 4.46 & 9.83 & 5.39 & 1 & 11.84 & 13.01 & 1 & 6.79 & 14.16 & 1 \\
\hline $60 \sim$ & 4.21 & 5.53 & 1 & 7.63 & 8.45 & 11.86 & 8.29 & 1 & 1 & 11.48 & 22.32 & 12.81 & 1 & 22.75 & 20.44 & 1 & 11.93 & & 1 \\
\hline
\end{tabular}

an increasing tendency in the control of diabetes from 2001 to 2008 , whereas the trend of control from 2008 to 2011 was similar to that of the treatment (Figure 2(b)).

Five studies reported the control of diabetes stratified by gender. Overall, the control rate of diabetes in males was $19.26 \%$ and was $19.03 \%$ for females. There was no significant difference between males and females $(\mathrm{OR}=1.06,95 \% \mathrm{CI}=$ 0.98-1.15) (Table 3).

The control rate of diabetes in urban areas was $16.7 \%$ $(95 \% \mathrm{CI}=-2.39-35.8 \%)$ and $18.59 \%(95 \% \mathrm{CI}=-1.2-38.39)$ in rural locations. No significant differences were found between urban and rural populations $(\mathrm{OR}=18.59,95 \% \mathrm{CI}=$ $-1.20-38.39)$ (Table 3).

\section{Discussion}

Due to the rapid development of the economy in China, the dietary habits and lifestyles of individuals have changed remarkably, which might have contributed to the increase in many chronic diseases such as diabetes $[11,80]$. Overall, the results of our study showed that the prevalence, awareness, treatment, and control of diabetes in mainland China were $6.41 \%, 45.81 \%, 42.54 \%$, and $20.87 \%$, respectively. A higher prevalence of diabetes was found in urban areas than in rural locations. As for the awareness of diabetes, this was also higher among residents in urban than in rural areas. There were no significant differences when data were stratified by gender or location in the subgroup analysis with regard to the treatment and control of diabetes. To the best of our knowledge, our study is the first systematic review on the awareness, treatment, and control of the diabetes and covers the longest time period from 1979 to 2012 in mainland China on the level of survey above the provincial.

The diabetes prevalence in our study ranged from $0.61 \%$ [27] to $20.85 \%$ [69] with an average of $6.41 \%$; however, many authors have shown different findings when studying other countries in Asia. Similar to our results, it was reported that the prevalence of diabetes in Indonesia is $5.7 \%$. In contrast, a survey conducted in Vietnam indicated a prevalence of $1.4 \%$ to $2.5 \%$, which is relatively lower than that of our study [81]. On the contrary, epidemiological data from Thailand and
Japan show that diabetes prevalence estimates were approximately $11.9 \%$ and $10 \%$, which were higher than our study [ 82 , 83]. The wide variation in the prevalence could be explained by the heterogeneity of the studies, geographical differences, and the application of different diagnostic and sampling methods. According to the statistics of the IDF, diabetes affects about 100 million people worldwide. By 2007, the number of affected individuals had reached 246 million [84]. From 1979 to 2002, nationwide epidemiological surveys of diabetes were conducted in China and the prevalence showed a 5-fold increase [27, 32, 85-87]. In accordance with this, an increasing trend in the prevalence of diabetes was found in our study. However, limited studies were included for pooled prevalence estimates; therefore, more studies with high quality and larger sample sizes are required to further confirm our findings.

As to the prevalence by gender, no significant difference could be found in our study between males and females, which is similar to the results of most studies according to the International Diabetes Federation (IDF) [84]. However, the study reported by Yang et al. in 2010 [72] showed a difference in the prevalence of diabetes between males and females. This might partly be explained by the fact that men are more accustomed to unhealthy diets due to their work and also smoke more frequently than women, which are the main risk factors for diabetes. Moreover, men are much more careless with regard to their health. However, further studies are required to confirm our findings.

In the analysis stratified by location, the prevalence of diabetes in urban areas was higher than in rural locations, with an odds ratio of 1.61 for residents in urban and rural areas, which suggests that residents in urban areas were more likely to suffer from diabetes than those in rural regions. In accordance with our findings, many studies worldwide have also reported a higher prevalence of diabetes in urban areas than in rural populations $[32,86,88]$. With the rapid development of the economy, urbanization has become a current trend in developing countries [89]. Urbanization is related to the change in the food supply and lifestyles which could lead to an unhealthy diet, sedentary habits, and overnutrition [72, 90]. The prevalence of overweight and obesity has increased 
TABLE 3: Overall awareness, treatment, and control of diabetes and subgroup analysis.

\begin{tabular}{|c|c|c|c|c|c|c|}
\hline \multicolumn{7}{|c|}{ Awareness } \\
\hline \multirow{2}{*}{ Overall awareness } & The number of studies & Case & Population & Awareness rate (\%) & $95 \% \mathrm{CI}$ & $I^{2}(\%)$ \\
\hline & 10 & 6,971 & 16,790 & 45.81 & $37.88 \sim 53.74$ & 98.8 \\
\hline \multicolumn{7}{|c|}{ Subgroup analysis } \\
\hline \multicolumn{7}{|l|}{ Gender } \\
\hline Male & 7 & 2,802 & 7,023 & 40.86 & $32.86 \sim 48.85$ & 97.0 \\
\hline Female & 7 & 3,126 & 7,822 & 41.58 & $31.47 \sim 51.70$ & 98.5 \\
\hline \multicolumn{7}{|l|}{ Location } \\
\hline Urban & 4 & 2,429 & 5,231 & 44.25 & $32.60 \sim 55.90$ & 98.0 \\
\hline Rural & 4 & 2,037 & 6,284 & 34.27 & $21.00 \sim 47.54$ & 98.5 \\
\hline \multicolumn{7}{|c|}{ Treatment } \\
\hline \multirow{2}{*}{ Overall treatment } & The number of studies & Case & Population & Treatment rate (\%) & $95 \% \mathrm{CI}$ & $I^{2}(\%)$ \\
\hline & 8 & 10,720 & 14,962 & 42.54 & $13.69 \sim 71.38$ & 99.9 \\
\hline \multicolumn{7}{|c|}{ Subgroup analysis } \\
\hline \multicolumn{7}{|l|}{ Gender } \\
\hline Male & 6 & 4,715 & 6,340 & 38.48 & $0.64 \sim 76.32$ & 99.9 \\
\hline Female & 6 & 5,117 & 6,976 & 41.18 & $6.96 \sim 75.40$ & 99.9 \\
\hline \multicolumn{7}{|l|}{ Location } \\
\hline Urban & 3 & 3,649 & 4,448 & 50.18 & $31.22 \sim 100.39$ & 99.8 \\
\hline Rural & 3 & 4,680 & 5,538 & 45.42 & $-12.7 \sim 103.60$ & 99.9 \\
\hline \multicolumn{7}{|c|}{ Control } \\
\hline \multirow{2}{*}{ Overall control } & The number of studies & Case & Population & Control rate (\%) & $95 \%$ CI & $I^{2}(\%)$ \\
\hline & 8 & 922 & 14,620 & 20.87 & $10.76 \sim 30.97$ & 99.5 \\
\hline \multicolumn{7}{|c|}{ Subgroup analysis } \\
\hline \multicolumn{7}{|l|}{ Gender } \\
\hline Male & 5 & 1,737 & 5,784 & 19.26 & $4.08 \sim 34.45$ & 99.4 \\
\hline Female & 5 & 1,777 & 6,085 & 19.03 & $4.73 \sim 33.33$ & 99.4 \\
\hline \multicolumn{7}{|l|}{ Location } \\
\hline Urban & 3 & 1,320 & 4,448 & 16.70 & $-2.39 \sim 35.80$ & 99.5 \\
\hline Rural & 3 & 1,809 & 5,538 & 18.59 & $-1.20 \sim 38.39$ & 99.4 \\
\hline
\end{tabular}

over the past two decades, especially in urban areas and highincome groups, which are the risk factors for diet-related disease and contribute to the development of diabetes [80].

Age is an important risk factor for the development of diabetes [91-93]. It was reported that the prevalence of diabetes increased with age $[85,86]$. A nationwide epidemiology survey showed that prevalence of diabetes in the age group of $20-39$ was $1.01 \%$, while it reached $6.31 \%$ in the age group over 40 ; however, the highest prevalence of $11.34 \%$ could be found in the age group over 60 [85]. Results of our study further confirm these findings. With the largest population in the world, China has become an ageing country; therefore, the prevalence of diabetes will increase as a result of the aging population in China. The health care for the aged population needs more attention from the government.

Ten studies provided information on the awareness of diabetes. Among the included study subjects, $45.81 \%$ were aware of having diabetes. From 1998 to 2011, there was no obvious improvement in the awareness rate of diabetes. However, we report a difference between the urban and rural areas when conducting stratified analysis by location. This difference in awareness may be a result of a lack of access to health care and knowledge in rural areas. Increasing the awareness through health education remains an effective method for the prevention and treatment of diabetes.

Among the participants who had diabetes in our study, $42.54 \%$ of them were undergoing treatment. This is high compared with the study which first reported the treatment rate of diabetes (27.2\%) in China [94]. The results of our study also showed an increasing trend in the treatment rate of diabetes from 2001 to 2011. However, no gender or location disparity was observed. The treatment of diabetes is not merely related to public awareness of the disease. More importantly, diagnostic methods play an essential role in the treatment of diabetes. Convenient, economic, and effective detection tools could improve the treatment rate of this disease.

It was reported that controlling blood glucose at a normal level can prevent many diabetes-related complications, such as retinopathy, nephropathy, neuropathy, and macroangiopathy [95]. Our results indicated an increasing trend in the control of diabetes. Also, no gender or location differences were found in the subgroup analysis. The control rate of diabetes in our study is $20.87 \%$, which is higher than that reported from a previous study with a control rate of 9.7\% [94], but lower than that identified in another study (40.3\%) [96]. The control of diabetes can be influenced by many factors, for example, 
obesity, smoking, insufficient exercise, and genetic susceptibility. Therefore, diversity exists in the control rate of diabetes in different places. Regular, effective, and timely treatment is primary in diabetes control. Moreover, adjusting dietary habits, changing lifestyles, and giving up smoking are essential when taking the risk factors for diabetes into account.

Coory [97] stated that it was very difficult to avoid heterogeneity in a meta-analysis, and this is also true for the metaanalysis of data from epidemiologic studies due to methodological problems. There were several methodological problems that might help to explain the heterogeneity: (1) different degrees of urbanization and socioeconomic conditions existed; (2) the included studies were carried out in different cities at various time points; (3) large differences existed in the age range, sampling methods, sample sizes, and response rates; (4) different diagnostic criteria and diagnosis methods were used in studies, with other studies not mentioning them at all; and (5) not all of the included studies provided sufficient information on gender, location, and age for subgroup analysis.

There are some limitations of the current study which need to be highlighted. Firstly, the present study might have underestimated the true prevalence of diabetes, since some of the studies included in the present review only used fasting plasma glucose (FPG) levels to diagnose new cases of diabetes; also, some studies did not provide any information about the diagnostic methods. Thus, more accurate measurements in the detection of undiagnosed diabetes are necessary in future research, for example, the two-hour glucose tolerance test and the oral glucose tolerance test (OGTT). Secondly, in addition to the subgroups of gender, location, and age, we did not report any positive risk factors associated with the prevalence of diabetes and the awareness, treatment, and control of the diabetes (e.g., overweight, obesity, hypertension, and hyperlipidemia); thus, we were unable to assess the association between them. Thirdly, the age range is quite different among the studies included. The age distribution in the sample population could markedly affect the results, since some locations contain a large number of individuals $>40$ years old; therefore, the prevalence of diabetes in some studies is likely to be higher than in others. Fourthly, limited studies were included in our meta-analysis for the pooled estimates; for example, only one study was included for analysis in 2009 and 2012 as studies from that time are possibly yet to be published. This could also be seen with regard to the trends in awareness of diabetes in 2001 and in the treatment and control of diabetes in 2011. Therefore, more studies were required for further analysis. A further limitation of the present study is that information in the subgroup analysis of awareness, control, and treatment by gender, location, and age was limited; therefore, we did not perform the relevant trend analyses.

The study showed an increasing trend for the prevalence, treatment, and control of diabetes among the population of mainland China from 1979 to 2012, but not in the awareness. This review will help us understand the gaps in the current research, which is useful for investigators and health care providers regarding aboriginal health. Thus, urgent measures are needed to prevent the high prevalence of diabetes and to improve diabetes awareness, control, and treatment among the Chinese populations.

\section{Authors' Contribution}

Min-zhi Li, Li Su, and Bao-yun Liang contributed equally to this work.

\section{Acknowledgments}

This work was supported by grants from the National Natural Science Foundation of China (Grant no. 81260594), the significant scientific project of the Guangxi University of Chinese Medicine (no. ZD2012007), the science and technology project of Traditional Chinese Medicine, Guangxi (no. GZKZ1107), the significant scientific research foundation of the Guangxi Health Department (no. 200933), the significant scientific research foundation of the Guangxi Health Department (no. 2012047), and the education reform project of Guangxi Medical University (2013XJGB01).

\section{References}

[1] P. Zimmet and K. G. M. M. Alberti, "Global and societal implications of the diabetes epidemic," Nature, vol. 414, no. 6865, pp. 782-787, 2001.

[2] A. A. Motala, M. A. K. Omar, and F. J. Pirie, "Epidemiology of type 1 and type 2 diabetes in africa," Journal of Cardiovascular Risk, vol. 10, no. 2, pp. 77-83, 2003.

[3] M. M. Engelgau, L. S. Geiss, J. B. Saaddine et al., "The evolving diabetes burden in the United States," Annals of Internal Medicine, vol. 140, no. 11, pp. 945-950, 2004.

[4] H. King, R. E. Aubert, and W. H. Herman, "Global burden of diabetes, 1995-2025: prevalence, numerical estimates, and projections," Diabetes Care, vol. 21, no. 9, pp. 1414-1431, 1998.

[5] S. Wild, G. Roglic, A. Green, R. Sicree, and H. King, "Global Prevalence of Diabetes: estimates for the year 2000 and projections for 2030," Diabetes Care, vol. 27, no. 5, pp. 1047-1053, 2004.

[6] B. Jönsson, "Revealing the cost of type II diabetes in Europe," Diabetologia, vol. 45, no. 7, pp. 5-12, 2002.

[7] P. Ricordeau, A. Weill, N. Vallier et al., “The prevalence and cost of diabetes in metropolitan France: what trends between 1998 and 2000?" Diabetes and Metabolism, vol. 29, no. 5, pp. 497-504, 2003.

[8] L. Ettaro, T. J. Songer, P. Zhang, and M. M. Engelgau, "Costof-illness studies in diabetes mellitus," PharmacoEconomics, vol. 22, no. 3, pp. 149-164, 2004.

[9] American Diabetes Association, "Economic costs of diabetes in the U.S. in 2007," Diabetes Care, vol. 31, no. 3, pp. 576-615, 2008.

[10] T. M. Dall, S. E. Mann, Y. Zhang et al., "Distinguishing the economic costs associated with type 1 and type 2 diabetes," Population Health Management, vol. 12, no. 2, pp. 103-110, 2009.

[11] C. Pan, "Diabetes care in China: meeting the challenge," World Hospitals and Health Services, vol. 41, no. 3, pp. 29-32, 2005.

[12] World Health Statistics, 2008, http://www.managediabetesnaturally.com/.

[13] B. Y. Pan, F. Fang, B. F. Luo, W. J. Liu, and J. Chen, “The prevalence and risk factors of diabetes mellitus in residents of Dongshan and Yueyang District of Guangzhou," Modern Preventive Medicine, vol. 32, pp. 516-517, 2005.

[14] A. D. Deshpande, M. Harris-Hayes, and M. Schootman, "Epidemiology of diabetes and diabetes-related complications," Physical Therapy, vol. 88, no. 11, pp. 1254-1264, 2008. 
[15] American Diabetes Association, "Diagnosis and classification of diabetes mellitus," Diabetes Care, vol. 33, supplement 1, pp. S62-S69, 2010.

[16] N. Sarwar, P. Gao, S. Seshasai et al., "Diabetes mellitus, fasting blood glucose concentration, and risk of vascular disease: a collaborative meta-analysis of 102 prospective studies," The Lancet, vol. 375, no. 9733, pp. 2215-2222, 2010.

[17] S. R. K. Seshasai, S. Kaptoge, A. Thompson et al., "Diabetes mellitus, fasting glucose, and risk of cause-specific death," The New England Journal of Medicine, vol. 364, no. 9, pp. 829-841, 2011.

[18] CDCP, National Diabetes Fact Sheet: General Information and National Estimates on Diabetes in the United States, 2005, US Department of Health and Human Services, Centers for Disease Control and Prevention, 2008.

[19] N. Poolsup, N. Suksomboon, and W. Setwiwattanakul, "Efficacy of various antidiabetic agents as add-on treatments to metformin in type 2 diabetes mellitus: systematic review and metaanalysis," ISRN Endocrinology, vol. 2012, Article ID 798146, 11 pages, 2012.

[20] C. L. Gillies, K. R. Abrams, P. C. Lambert et al., "Pharmacological and lifestyle interventions to prevent or delay type 2 diabetes in people with impaired glucose tolerance: systematic review and meta-analysis," British Medical Journal, vol. 334, no. 7588, pp. 299-302, 2007.

[21] D. Hu, P. Fu, J. Xie et al., "Increasing prevalence and low awareness, treatment and control of diabetes mellitus among Chinese adults: the InterASIA study," Diabetes Research and Clinical Practice, vol. 81, no. 2, pp. 250-257, 2008.

[22] S. Lv, X. Pan, Q. Xiang, and M. Wu, "Analysis on the level of plasma glucose and prevalence of diabetes among residents of Jiangsu province," Jiangsu Journal of Preventive Medicine, vol. 22, no. 1, pp. 10-12, 2011.

[23] Y.-N. Yang, X. Xie, Y.-T. Ma et al., “Type 2 diabetes in xinjiang uygur autonomous region, china," PLoS One, vol. 7, no. 4, Article ID e35270, 2012.

[24] H. Li, B. Oldenburg, C. Chamberlain et al., "Diabetes prevalence and determinants in adults in China mainland from 2000 to 2010: a systematic review," Diabetes Research and Clinical Practice, vol. 98, no. 2, pp. 226-235, 2012.

[25] The Nordic Cochrane Centre, "The Cochrane Collaboration. Review Manager (RevMan). 5. 1. Copenhagen: the Nordic Cochrane Centre, The Cochrane Collaboration," 2011.

[26] J. P. T. Higgins, S. G. Thompson, J. J. Deeks, and D. G. Altman, "Measuring inconsistency in meta-analyses," British Medical Journal, vol. 327, no. 7414, pp. 557-560, 2003.

[27] National Diabetes Research Group, "Diabetes mellitus survey of 300,000 in fourteen provinces and cities of China," Chinese Medical Journal, vol. 20, pp. 678-681, 1981.

[28] CDC, "Report on Chronic Disease Risk Factor Surveillance in China 2010," 2012.

[29] S. Sha and Z. Wang, "Diabetes mellitus survey of 100,000 in Shanghai area," Shanghai Medical Journal, vol. 3, article 012, 1980.

[30] H. Xiang, D. Wang, and Q. Sun, "A survey of diabetes and impaired glueose toleranee in Shanxi, Beijing and Liaoning, north China 1987," Chinese Journal of Diabetes, vol. 1, no. 1, p. $16,1993$.

[31] H. Xu and X. Zhu, "An epidemiological study on diabetes mellitus in Yunnan province," Railway Medical Journal, vol. 4, 1996.
[32] X. Pan, W. Yang, and J. Liu, "Prevalence of diabetes and its risk factors in China 1994. National Diabetes Prevention and Control Cooperative Group," Chinese Journal of Internal Medicine, vol. 36, no. 6, pp. 384-389, 1997.

[33] M. Yang, W. Mo, C. Wang et al., "An epidemiological study on diabetes mellitus in Anhui Province," Journal of Disease Control \& Prevention, vol. 2, 1997.

[34] Z. Zhang, "Comparative report of two Investigatios for diabetes in Fujian Province," The Journal of Fujian Medical University, vol. 31, no. 2, pp. 229-231, 1997.

[35] T. Liu and B. Zhang, "Epidemiological studies on diabetes," Ningxia Medical Journal, vol. 20, no. 1, pp. 22-24, 1998.

[36] H. Xiang, W. Wu, C. Liu et al., "A epidemiological study on diabetes mellitus 1995-1996, in China," Chinese Journal of Diabetes, vol. 3, pp. 131-133, 1998.

[37] X. Zhang, "Epidemiological studies on diabetes in Heibei," Practice Preventive Medicine, vol. 2, 1998.

[38] Z. Zhao, "Study on the prevalence and risk factors of diabetes mellitus in Shandong Province," Chinese Journal of Disease Control \& Prevention, vol. 6, no. 1-6, pp. 280-282, 1998.

[39] Z.-Z. Wang, X.-Z. Huang, S.-B. Tang et al., "The prevalence of NIDDM and IGT and related factors among residents in some areas of hubei province, China," Biomedical and Environmental Sciences, vol. 13, no. 2, pp. 148-153, 2000.

[40] Z. Sheng, M. Liu, Y. Wang et al., "A survey of diabetes prevalence in 9376 urbanite adults or Shanghai," Chinese Journal of Diabetes, vol. 4, article 006, 2001.

[41] S. Wang, C. Wu, and H. Deng, "A cross-sectional study on diabetes mellitus in Guangdong Province," Guangdong Medical Journal, vol. 22, no. 6, pp. 455-458, 2001.

[42] G. Liu and Z. Feng, "Investigation on the epidemiological characteristics of diabetes mellitus and impaired glucose tolerance in natural population of Henan Province," Journal of Zhengzhou University, vol. 37, no. 4, pp. 499-501, 2002.

[43] K. Gu, F. Shi, X. Li et al., "Investigation on prevalence of main chronic diseases in Shanghai," Chinese Journal Preventive Control Crohn's Disease, vol. 12, no. 3, pp. 116-136, 2004.

[44] X. Ma, Z. Li, L. Zou, and H. Liu, "A cross-sectional study on diabetes mellitus in Qinghai Province," Qinghai Medical Journal, vol. 34, no. 2, pp. 57-59, 2004.

[45] X. Li, Y. Yi, C. Feng et al., "Investigation and analysis on the suffering status of chronic disease in urban and rural residents of Sichuan Province," Journal of Preventive Medicine Information, vol. 2, article 003, 2005.

[46] L. Wang, "Chinese residents nutrition and health survey reportcomprehensive report 2002," 2005.

[47] R. Hu, M. Yu, J. Zhong, and W. Gong, "The changing trends of the incidences and the risk factors of chronic diseases among residents in the cities and rura areas in Zhejiang Province," Disease Surveillance, vol. 21, no. 9, pp. 480-483, 2006.

[48] J. Z. Jixin Sun, J. Zhang, Y. Xue, J. Zhang, L. Wang, and Y. Cao, "Surveillance on prevalence of diabetes among adults in Hebei province," Chinese Journal of Public Healt, vol. 22, pp. 801-803, 2006.

[49] R. Li, W. Lu, W. Jia et al., "Cross-sectional investigation of prevalence of type 2 diabetes in Shanghai," Zhonghua yi xue za zhi, vol. 86, no. 24, p. 1675, 2006.

[50] R. Li, W. Lu, W.-P. Jia et al., "Cross-sectional investigation of prevalence of type 2 diabetes in Shanghai," National Medical Journal of China, vol. 86, no. 24, pp. 1675-1680, 2006. 
[51] P.-H. Zhang, S.-J. Jiao, Y. Zhou et al., "Studies on prevalence and control of several common chronic diseases among Beijing adults in 2005," Zhonghua liu xing bing xue za zhi, vol. 28, no. 7, pp. 625-630, 2007.

[52] S. Zhao, X. Chen, Y. Wang et al., "The investigation of prevaIence and risk factors of diabetes mellitus in Shandong coastal area," Chinese Journal of Diabetes, vol. 15, no. 12, pp. 729-732, 2007.

[53] C. Fan, M. Yu, and Y. Chen, "Prevalence and risk factors of type II diabetes mellitus in residents of Zhejiang Province," Zhejiang Journal of Preventive Medicine, vol. 3, article 003, 2008.

[54] L. Xu, X. Xie, S. Wang et al., "Prevalence of diabetes mellitus in China," Experimental and Clinical Endocrinology \& Diabetes, vol. 116, no. 1, p. 69, 2008.

[55] Y. Yu, A. Xu, J. Ma et al., "Analysis on epidemiologic characteristics of diabetes mellitus and impaired glucose regulation among rural residents in Shandong Province, China," Chinese Journal of Public Health, vol. 24, no. 12, pp. 1454-1456, 2008.

[56] M. Cheng, S. Miao, and X. Li, "An investigation on prevalence of chronic diseases, and the awareness and the demands for healthcare in residents aged $>18$ years in Shanghai Communities," Chinese Journal Preventive Control Crohn's Disease, no. 2, pp. 194-197, 2009.

[57] X. W. Gengle Zhi, X. Liu, G. Song, H. Zu, F. Wang, and J. Wang, "A study on prevalence of type 2 diabetes in Tianjin," Tianjin Medical Journal, vol. 37, pp. 101-103, 2009.

[58] L. Lin, G. Chen, X. Zou et al., "Diabetes, pre-diabetes and associated risks on Minnesota code-indicated major electrocardiogram abnormality among Chinese: a cross-sectional diabetic study in Fujian province, southeast ChinaComplications," Obesity Reviews, vol. 10, no. 4, pp. 420-430, 2009.

[59] H. Tian, G. Song, H. Xie, H. Zhang, J. Tuomilehto, and G. Hu, "Prevalence of diabetes and impaired fasting glucose among 769 792 rural Chinese adults," Diabetes Research and Clinical Practice, vol. 84, no. 3, pp. 273-278, 2009.

[60] H. Wang, Q. Qiu, L.-L. Tan et al., "Prevalence and determinants of diabetes and impaired fasting glucose among urban community-dwelling adults in Guangzhou, China," Diabetes and Metabolism, vol. 35, no. 5, pp. 378-384, 2009.

[61] H. Zhang, J. Gao, Q. Dai et al., "A Cross-sectional study on diabetes mellitus in partial areas of Qinghai Province," Journal of High Altitude Medicine, no. 2, pp. 11-14, 2009.

[62] S. Zhang, W. Tong, T. Xu, B. Wu, and Y. Zhang, "Diabetes and impaired fasting glucose in Mongolian population, Inner Mongolia, China," Diabetes Research and Clinical Practice, vol. 86, no. 2, pp. 124-129, 2009.

[63] S. Zhao, Y. Hou, and H. Rao, "Cross-sectional study on diabetes mellitus among urban and rural residents in Shanxi Province," China Preventive Medicine, vol. 1, article 002, 2009.

[64] X. Chen, H. Bo, P. Huang et al., "Prevalence of metabolic syndrome incommunity elderly adults of Shanghai city," Chinese Journal of Public Health, vol. 26, no. 4, pp. 498-499, 2010.

[65] Y. Dai, B. Yuan, and Z. Shi, "A Cross-sectional study on diabetes mellitus among adults of 18 years old and above in Jiangsu Province," Journal of Hygiene Research, vol. 39, no. 5, pp. 626-623, 2010.

[66] N. Jin, J. Dou, S. Wang et al., "A survey of prevalence of diabetes mellitus and metabolic syndrome in Beijing," Chinese Journal of Diabetes, vol. 2, no. 6, 2010.
[67] Q. L. Kaiting Chen, N. Wu, Y. Feng, X. Liu, S. Li, and J. Zhang, "Analysis on relationship between abnormal glucose metabolism and age, gender of residents in Heilongjiang province," Chinese Journal of Diabetes Mellitus, vol. 2, pp. 419-423, 2010.

[68] J. B. Li, X. Wang, J.-X. Zhang et al., "Metabolic syndrome: prevalence and risk factors in Southern China," Journal of International Medical Research, vol. 38, no. 3, pp. 1142-1148, 2010.

[69] N. Su, Y. Wang, Q. Chen et al., "Community management of patients with hypertension and diabetes in Beijing," Chinese Journal of Public Health, no. 7, pp. 900-901, 2010.

[70] J. Wei, F. Liu, L. Zhou et al., "An epidemiological survey of risk factors on diabetes and impaired fasting glucose and traditional Chinese medicine syndrome in Beijing," Beijing Journal of Traditional Chinese Medicine, vol. 29, no. 010, pp. 731-737, 2010.

[71] W. Wei, S.-Y. Liu, F.-F. Zeng et al., “Type 2 diabetes and impaired glucose tolerance in North-China-based rural community adults," Public Health, vol. 124, no. 10, pp. 593-601, 2010.

[72] W. Yang, J. Lu, J. Weng et al., "Prevalence of diabetes among men and women in China," The New England Journal of Medicine, vol. 362, no. 12, pp. 1090-1101, 2010.

[73] C. Le, D. Jun, S. Zhankun, L. Yichun, and T. Jie, "Socioeconomic differences in diabetes prevalence, awareness, and treatment in rural southwest China," Tropical Medicine and International Health, vol. 16, no. 9, pp. 1070-1076, 2011.

[74] X. Qi, J. Sun, J. Wang et al., "Prevalence and correlates of latent autoimmune diabetes in adults in Tianjin, China: a populationbased cross-sectional study," Diabetes Care, vol. 34, no. 1, pp. 6670, 2011.

[75] Z. Ye, L. Cong, G. Ding et al., "A survey of the prevalence of diabetes mellitus in adults of Zhejiang Province," Chinese Journal of Endocrinology and Metabolism, vol. 27, no. 12, pp. 988-991, 2011.

[76] X. Chen, X. Guo, Z. Lu et al., "Regression analysis on risk factors of impaired glucose regulation and diabetes mellitus among residents of Shandong Province," Chinese Journal of Public Health, vol. 28, no. 11, pp. 1403-1407, 2012.

[77] R. Li, W. Lu, Q. W. Jiang et al., "Increasing prevalence of type 2 diabetes in Chinese adults in Shanghai," Diabetes Care, vol. 35, no. 5, pp. 1028-1030, 2012.

[78] Q. Liu, P. Liang, L. Xu et al., "Epidemiology research of diabetic retinopathy in Ningxia region," International Journal of Ophthalmology, vol. 12, no. 8, pp. 1566-1569, 2012.

[79] Y. Zhan, J. Yu, D. Hu et al., "Prevalence and related knowledge of diabetes mellitus among residents of Beijing," Chinese Journal of Public Health, vol. 28, no. 1, pp. 19-21, 2012.

[80] Y. Wang, J. Mi, X.-Y. Shan, Q. J. Wang, and K.-Y. Ge, "Is China facing an obesity epidemic and the consequences? The trends in obesity and chronic disease in China," International Journal of Obesity, vol. 31, no. 1, pp. 177-188, 2006.

[81] P. S. Q. Phan Si Quoc, M.-A. Charles, N. H. C. Nguyen Huy Cuong et al., "Blood glucose distribution and prevalence of diabetes in Hanoi (Vietnam)," American Journal of Epidemiology, vol. 139, no. 7, pp. 713-722, 1994.

[82] Y. Akazawa, "Prevalence and incidence of diabetes mellitus by WHO criteria," Diabetes Research and Clinical Practice, vol. 24, pp. S23-S27, 1994.

[83] C. S. Cockram, "The epidemiology of diabetes mellitus in the Asia-Pacific region," Hong Kong Medical Journal, vol. 6, no. 1, pp. 43-52, 2000.

[84] IDF, Diabetes Atlas, International Diabetes Federation, Brussels, Belgium, 3rd edition, 2006. 
[85] K. Wang, T. Li, and H. Xiang, "Study on the epidemiological characteristics of diabetes mellitus and IGT in China," Chinese Journal of Epidemiology, vol. 19, no. 5, pp. 282-285, 1998.

[86] D. Gu, K. Reynolds, X. Duan et al., "Prevalence of diabetes and impaired fasting glucose in the Chinese adult population: international Collaborative Study of Cardiovascular Disease in Asia (InterASIA)," Diabetologia, vol. 46, no. 9, pp. 1190-1198, 2003.

[87] L. Li, K. Rao, and L. Kong, "A description on the Chinese national nutrition and health survey in 2002," Chinese Journal of Infection Epidemiology, vol. 26, no. 7, pp. 478-484, 2005.

[88] R. B. Singh, S. Bajaj, M. A. Niaz, S. S. Rastogi, and M. Moshiri, "Prevalence of type 2 diabetes mellitus and risk of hypertension and coronary artery disease in rural and urban population with low rates of obesity," International Journal of Cardiology, vol. 66, no. 1, pp. 65-72, 1998.

[89] B. Cohen, "Urbanization in developing countries: current trends, future projections, and key challenges for sustainability," Technology in Society, vol. 28, no. 1-2, pp. 63-80, 2006.

[90] J. C. N. Chan, V. Malik, W. Jia et al., "Diabetes in Asia: epidemiology, risk factors, and pathophysiology," Journal of the American Medical Association, vol. 301, no. 20, pp. 2129-2140, 2009.

[91] N. T. Artinian, M. A. Nies, S. Myers Schim, J. S. Vander Wal, and M. K. Keves-Foster, "Hypertension and diabetes in Detroit Hispanics," Applied Nursing Research, vol. 17, no. 3, pp. 158-167, 2004.

[92] P. O. Ayanbadejo, K. O. Savage, and S. O. Jeboda, "Awareness of periodontal diseases amongst Nigerian diabetics," Tropical Dental Journal, vol. 27, no. 105, pp. 13-16, 2004.

[93] P. Namperumalsamy, R. Kim, K. Kaliaperumal, A. Sekar, A. Karthika, and P. K. Nirmalan, "A pilot study on awareness of diabetic retinopathy among non-medical persons in South India. The challenge for eye care programmes in the region," Indian Journal of Ophthalmology, vol. 52, no. 3, pp. 247-251, 2004.

[94] Y.-F. Wu, G.-Q. Xie, Y. Li, L.-C. Zhao, and B.-F. Zhou, “The current status on the prevalence, awareness, treatment and control of diabetes mellitus in several Chinese subpopulations," Chinese Journal of Epidemiology, vol. 26, no. 8, pp. 564-568, 2005.

[95] DCCT, "The effect of intensive treatment of diabetes on the development and progression of long-term complication in insul independent diabetes mellitus," The New England Journal of Medicine, vol. 329, pp. 977-986, 1993.

[96] X.-G. Li, Y.-S. Shi, S. Bai, J. Luo, and B. Zhao, "Survey on the awareness, treatment and control rates as well as the risk factors in patients with diabetes in the Bajing community of Shenyang city," Chinese Journal of Clinical Rehabilitation, vol. 9, no. 11, pp. 6-7, 2005.

[97] M. D. Coory, "Comment on: heterogeneity in meta-analysis should be expected and appropriately quantified," International Journal of Epidemiology, vol. 39, no. 3, p. 932, 2009. 


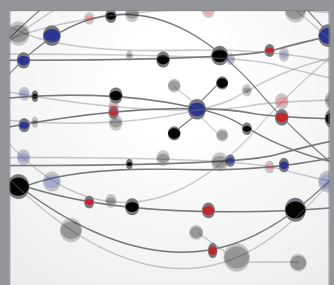

The Scientific World Journal
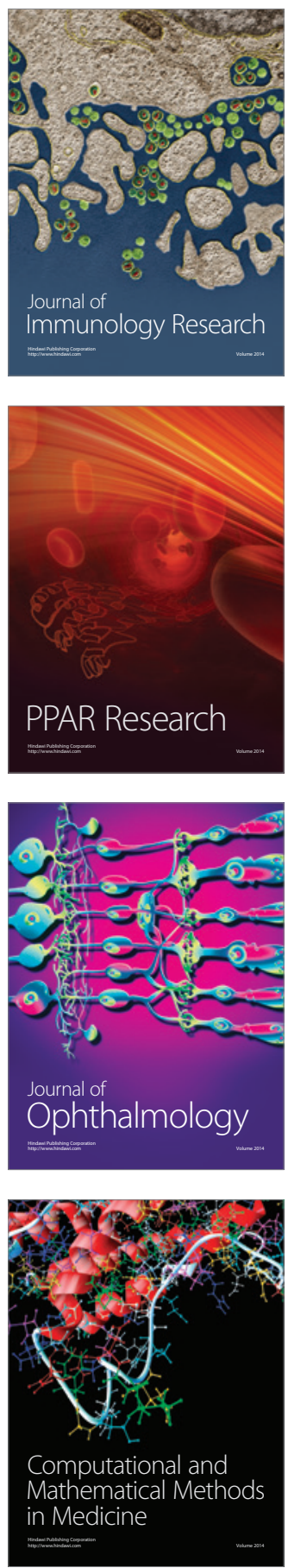

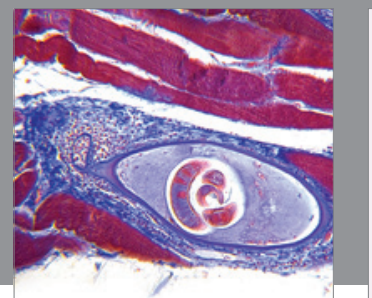

Gastroenterology

Research and Practice
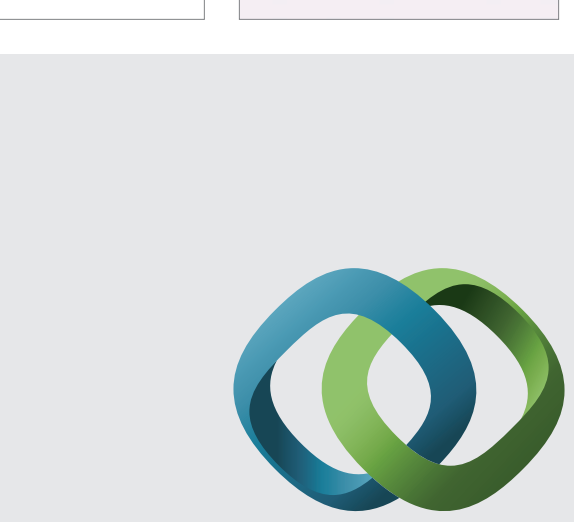

\section{Hindawi}

Submit your manuscripts at

http://www.hindawi.com
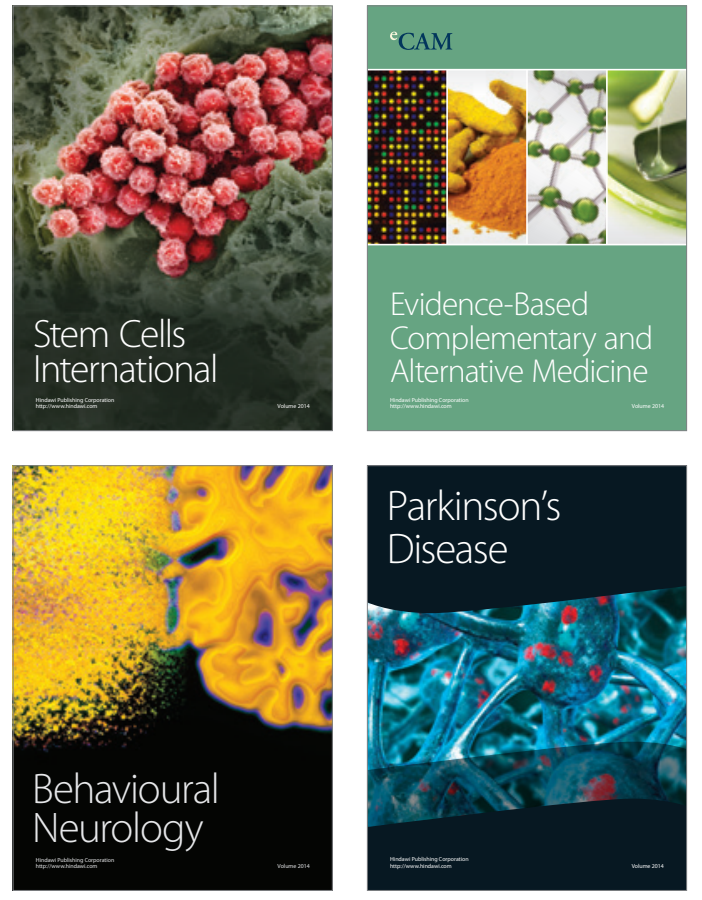
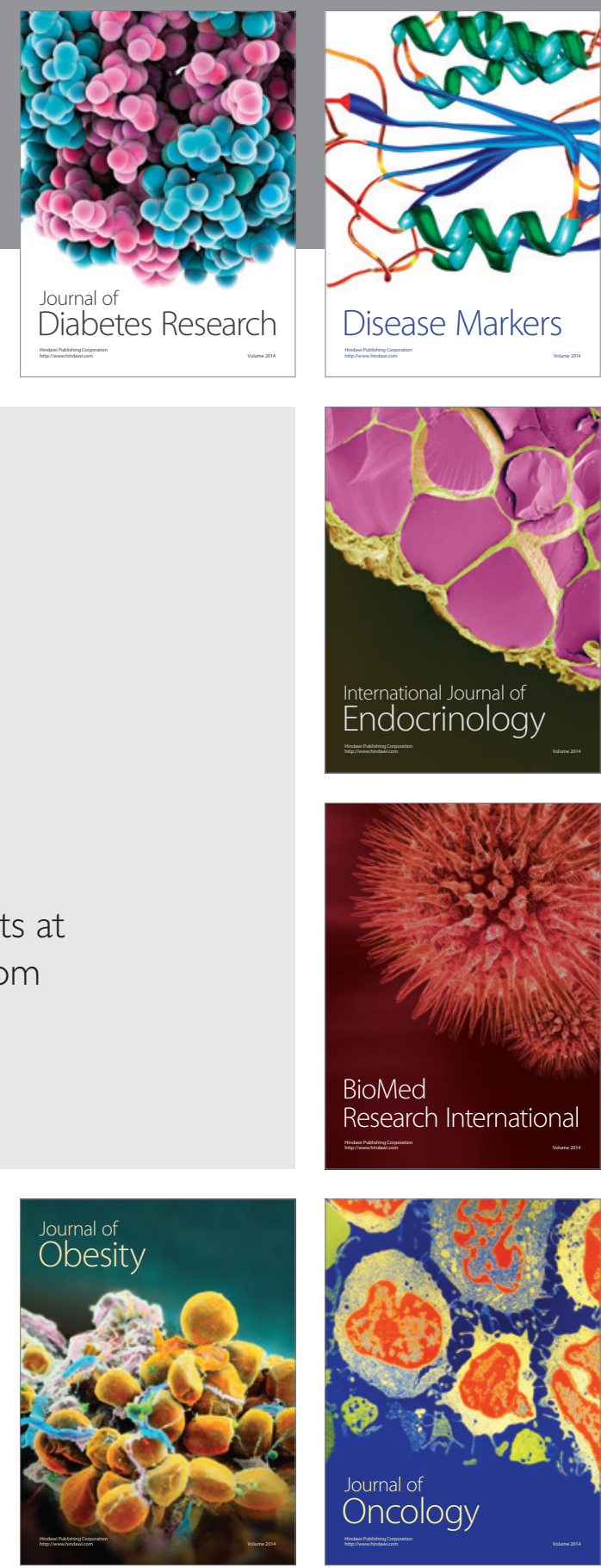

Disease Markers
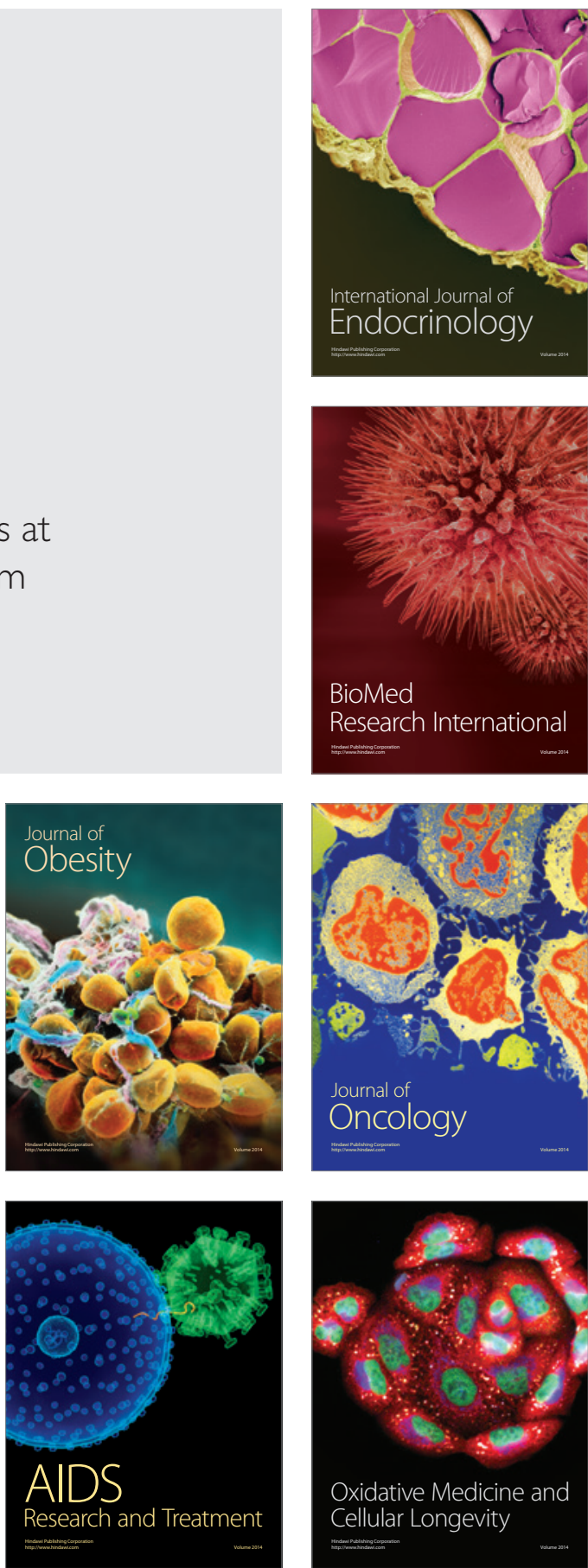